\title{
Article \\ Boundedness of Some Paraproducts on Spaces of Homogeneous Type
}

\author{
Xing Fu
}

check for

updates

Citation: Fu, X. Boundedness of Some Paraproducts on Spaces of Homogeneous Type. Mathematics 2021, 9, 2591. https://doi.org/ $10.3390 /$ math 9202591

Academic Editor: Jay Jahangiri

Received: 1 August 2021

Accepted: 11 October 2021

Published: 15 October 2021

Publisher's Note: MDPI stays neutral with regard to jurisdictional claims in published maps and institutional affiliations.

Copyright: (c) 2021 by the authors. Licensee MDPI, Basel, Switzerland. This article is an open access article distributed under the terms and conditions of the Creative Commons Attribution (CC BY) license (https:// creativecommons.org/licenses/by/ $4.0 /)$.
Hubei Key Laboratory of Applied Mathematics, Faculty of Mathematics and Statistics, Hubei University, Wuhan 430062, China; xingfu@hubu.edu.cn

Abstract: Let $(\mathcal{X}, d, \mu)$ be a space of homogeneous type in the sense of Coifman and Weiss. In this article, the author develops a partial theory of paraproducts $\left\{\Pi_{j}\right\}_{j=1}^{3}$ defined via approximations of the identity with exponential decay (and integration 1), which are extensions of paraproducts defined via regular wavelets. Precisely, the author first obtains the boundedness of $\Pi_{3}$ on Hardy spaces and then, via the methods of interpolation and the well-known $T(1)$ theorem, establishes the endpoint estimates for $\left\{\Pi_{j}\right\}_{j=1}^{3}$. The main novelty of this paper is the application of the Abel summation formula to the establishment of some relations among the boundedness of $\left\{\Pi_{j}\right\}_{j=1}^{3}$, which has independent interests. It is also remarked that, throughout this article, $\mu$ is not assumed to satisfy the reverse doubling condition.

Keywords: space of homogeneous type; paraproduct; $T(1)$ theorem; hardy space; bilinear estimate

\section{Introduction}

Classical paraproducts defined via convolutions are kinds of non-commutative bilinear operators, which are useful tools in the decompositions of products of functions. The prototypes of paraproducts can be found, for examples, in the work of Fujita and Kato [1] and Kato [2] on the study of mild solutions of Navier-Stokes equations and in the investigation of pseudo-differential operators and para-differential operators by Meyer and Coifman [3-5]. The formal notion of paraproducts has been introduced in 1981 by Bony for the study of the nonlinear hyperbolic partial differential equations in [6]. Since then the theory of papraproducts has been developed rapidly, which plays an essential role in both harmonic analysis and partial differential equations. For applications of paraproducts in harmonic analysis, we refer the reader to [7-16]. See also [17,18] for more applications of paraproducts in mathematical physics. The paraproducts defined via wavelets was first investigated by Grafakos and Torres [19] and then studied by Bonami et al. [20], which play crucial roles in both the bilinear decompositions of products of functions in $[20,21]$, the (sub-)bilinear decompositions of commutators and the endpoint estimates of commutators in $[22,23]$. See the survey [24] and the monographs [25,26] for more information.

In 1970s, Coifman and Weiss $[27,28]$ introduced the notion of the space of homogeneous type which has been proven to be a natural background for extensions of many classical results on Euclidean spaces. Recall that a quasi-metric space $(\mathcal{X}, d)$ is a non-empty set $\mathcal{X}$ equipped with a quasi-metric $d$ such that, for any $x, y, z \in \mathcal{X}$,

(i) $\quad d(x, y)=0$ if and only if $x=y$;

(ii) $d(x, y)=d(y, x)$;

(iii) the quasi-triangle inequality $d(x, y) \leq A_{0}[d(x, z)+d(z, y)]$ holds true, where $A_{0} \in$ $[1, \infty)$ is called the quasi-triangle constant which is independent of $x, y$ and $z$.

The triple $(\mathcal{X}, d, \mu)$ is called a space of homogeneous type if $\mu$ is a non-negative measure satisfying the following doubling condition: there exists a positive constant $C_{(\mathcal{X})} \in[1, \infty)$, depending on $\mathcal{X}$, such that, for any $r \in(0, \infty)$ and $x \in \mathcal{X}$,

$$
\mu(B(x, 2 r)) \leq C_{(\mathcal{X})} \mu(B(x, r))
$$


or, equivalently, there exists a positive constant $C$ such that, for any $\lambda \in[1, \infty), r \in(0, \infty)$ and $x \in \mathcal{X}$,

$$
\mu(B(x, \lambda r)) \leq C \lambda^{n} \mu(B(x, r)),
$$

where $B(x, r):=\{y \in \mathcal{X}: d(y, x)<r\}$ and $n:=\log _{2} C_{(\mathcal{X})}$ represents the "upper dimension" of $\mathcal{X}$.

As in ([29], Section 1) (see also ([30], Section 1)), throughout the whole article, we always assume that $(\mathcal{X}, d, \mu)$ is a space of homogeneous type satisfying the following additional assumptions:

(i) Suppose that, for any given $x \in \mathcal{X}$, the sequence of balls, $\{B(x, r)\}_{r \in(0, \infty)}$, in $\mathcal{X}$ is a basis of open neighborhoods around $x$.

(ii) Assume that $\mu$ satisfies that all the open sets are measurable and, for any measurable set $A \subset \mathcal{X}$, there exists a Borel set $E \supset A$ such that $\mu(A)=\mu(E)$, which is called Borel regular.

(iii) Suppose that, for any $x \in \mathcal{X}$ and $r \in(0, \infty), \mu(B(x, r)) \in(0, \infty)$.

(iv) For the sake of the presentation simplicity, without loss of generality, we always assume that $\operatorname{diam}(\mathcal{X}):=\sup \{d(x, y): x, y \in \mathcal{X}\}=\infty$ and $(\mathcal{X}, d, \mu)$ is non-atomic, that is, for any $x \in \mathcal{X}, \mu(\{x\})=0$.

It was shown in ([31], Lemma 5.1) or ([32], Lemma 8.1) (see also ([30], Section 1)) that, under the above assumptions, $\operatorname{diam}(\mathcal{X})=\infty$ if and only if $\mu(\mathcal{X})=\infty$.

A space of homogeneous type, $(\mathcal{X}, d, \mu)$, is called an RD-space introduced by Han et al. [33] (see also [34]) if $\mu$ further satisfies the following reverse doubling condition (or, for brevity, RD-condition): there exist positive constants $a_{0}, \widetilde{C}_{(\mathcal{X})} \in(1, \infty)$, depending on $\mathcal{X}$, such that, for any $x \in \mathcal{X}$ and $r \in\left(0, \operatorname{diam}(\mathcal{X}) / a_{0}\right)$,

$$
\mu\left(B\left(x, a_{0} r\right)\right) \geq \widetilde{C}_{(\mathcal{X})} \mu(B(x, r)) .
$$

Notice that the harmonic analysis on spaces of homogeneous type has a long history; see, for example, $[27,28,35,36]$. We refer the reader to [33,34,37-46] for the real-variable theory of some function spaces and Calderón-Zygmund operators on RD-spaces. Furthermore, for some recent developments on the real-variable theory of function spaces and its applications on spaces of homogeneous type, please see [29,47-61].

Some progress is also made on the boundedness of paraproducts on metric measure spaces. Let $(\mathcal{X}, d, \mu)$ be an RD-space. Han et al. ([33], Theorem 5.56) extended the celebrated T(1)-theorem of David and Journé [11] to the RD-space via paraproducts. Later, Grafakos et al. [43] introduced a kind of paraproducts on $\mathcal{X}$, which extends the corresponding notion of paraproducts in ([33], Theorem 5.56), and investigated their boundedness from $H^{p}(\mathcal{X}) \times H^{q}(\mathcal{X})$ into $H^{r}(\mathcal{X})$ by (in)homogeneous Calderón reproducing formulae, which also generalizes a classical result on Euclidean spaces obtained by Grafakos and Kalton [14]. Grafakos et al. [43] also studied the endpoint estimates of paraproducts on $\mathcal{X}$ via the theory of Calderón-Zygmund operators. Moreover, via the off-diagonal estimates of integral kernels, Grafakos et al. [42] showed that a kind of bilinear discrete paraproducts on $\mathcal{X}$ via the theory of multilinear Calderón-Zygmund operators established in [42], are bounded on weighted Lebesgue spaces, Triebel-Lizorkin spaces and Besov spaces. Recently, Chang et al. [30,62] showed that the aforementioned boundedness of paraproducts on RD-spaces remains true on spaces of homogeneous type, namely, without having recourse to the $\mathrm{RD}$-condition (2).

A space of homogeneous type, $(\mathcal{X}, d, \mu)$, is called a metric measure space of homogeneous type if the quasi-triangle constant $A_{0}=1$. In this setting, Fu et al. [48] proved that $f \times g$ of $f \in H_{\text {at }}^{1}(\mathcal{X})$ and $g \in \operatorname{BMO}(\mathcal{X})$ can be written into a sum of three bilinear operators $\left\{\Pi_{j}\right\}_{j=1}^{3}$, which are also called paraproducts. These paraproducts play important roles in the study on the endpoint boundedness of the (sub-)linear commutator $[b, T]$ of a (sub)linear operator $T$ and $b \in \operatorname{BMO}(\mathcal{X})$ on (local) Hardy spaces in $[29,57,58]$; see also the survey [63] for more details. A natural question is whether there exists a relatively complete 
boundedness theory for paraproducts $\left\{\Pi_{j}\right\}_{j=1}^{3}$ in [48] which enjoy the same boundedness as the paraproducts in $[30,62]$.

In this article, we give a partial affirmative answer to this question with the paraproducts $\left\{\Pi_{j}\right\}_{j=1}^{3}$ in [48] replaced by more general forms via the exp-ATIs and 1-exp-ATIs from [53]. We obtained the boundedness of $\Pi_{3}$ on Hardy spaces and its endpoint estimates, and the endpoint estimates for $\Pi_{1}$ and $\Pi_{2}$. The boundedness of $\Pi_{1}$ and $\Pi_{2}$ on Hardy spaces may need different approaches and was left as an unsolved question.

In what follows, we always assume that $(\mathcal{X}, d, \mu)$ is a space of homogeneous type. The remainder of this article is organized as follows.

Section 2 is devoted to some preliminary notions and results which are needed to the proof of the main results Theorems $2-4$ below. In particular, we recall the $T(1)$ theorem from ([32], Section 12) (see Lemma 3 below), and use the Abel summation formula to build some relations among the boundedness of $\left\{\Pi_{j}\right\}_{j=1}^{3}$ (see Theorem 1 below).

In Section 3, we prove Theorems $2-4$ below. In precise, Theorem 2 is an easy consequence of the Hölder inequality and the definition of $H^{p}(\mathcal{X})$. To show (i)-(iv) of Theorem 3, we first fix an $f \in \operatorname{BMO}(\mathcal{X})$ and express the paraproduct $\Pi_{3}$ by an integral operator $K_{f}^{(3)}$. Then, via the methods of interpolation and the crucial estimates (11) and (12), we show that $K_{f}^{(3)}$ has the weak boundedness property $\mathrm{WBP}(\eta)$ with $\eta$ as in Lemma 2 below. Next we prove that the kernel of $K_{f}^{(3)}$ is an $\eta$-Calderón-Zygmund kernel, which also relies on estimates (11) and (12). Moreover, we point out that $K_{f}^{(3)}(1),\left(K_{f}^{(3)}\right)^{*}(1) \in \mathrm{BMO}(\mathcal{X})$, which, together with the $T(1)$ theorem from ([32], Theorem 12.2) and the boundedness of Calderón-Zygmund operators, we finally finish the proof of (i)-(iv) of Theorem 3. In order to prove (v) and (vi) of Theorem 3, we first fix $g \in L^{\infty}(\mathcal{X})$ and write $\Pi_{3}$ as an integral operator $K_{g}^{(3)}$. By the fact that $L^{\infty}(\mathcal{X}) \subset \operatorname{BMO}(\mathcal{X})$ and some arguments used in the proof of (i)-(iv) of Theorem 3, we obtain the desired results and finish the proof of Theorem 3. The proof of (i)-(iv) of Theorem 4 is a consequence of the arguments and ideas from the proof of (i)-(iv) of Theorem 3. The main novelty of this paper lies in the proof of (v)(vi) of Theorem 4, where we use the Abel summation formula to build some relations among the boundedness of $\left\{\Pi_{j}\right\}_{j=1}^{3}$ and then transform the same boundedness of $\Pi_{1}$ from $L^{2}(\mathcal{X}) \times L^{\infty}(\mathcal{X})$ into $L^{2}(\mathcal{X})$ into the same boundedness of $\Pi_{2}$ and $\Pi_{3}$. We also remark that, throughout this article, $\mu$ is not assumed to satisfy the reverse doubling condition (2).

Finally, we list some notation used throughout this article. Let $\mathbb{N}:=\{1,2, \ldots\}$ and $\mathbb{Z}_{+}:=\{0\} \cup \mathbb{N}$. We use $C$ or $c$ to denote a positive constant which may be different from line to line, but is independent of main parameters. In addition, we also use $C_{(\rho, \alpha, \ldots)}$ or $c_{(\rho, \alpha, \ldots)}$ to denote a positive constant depending on the indicated parameters $\rho, \alpha, \ldots$ For any two real functions $f$ and $g$, we write $f \lesssim g$ when $f \leq C g$ and $f \sim g$ when $f \lesssim g \lesssim f$. For any subset $E$ of $\mathcal{X}$, denote by $\mathbf{1}_{E}$ its characteristic function. For any $x, y \in \mathcal{X}$, $r, \rho \in(0, \infty)$ and ball $B:=B(x, r):=\{y \in \mathcal{X}: d(y, x)<r\}$, define $\rho B:=B(x, \rho r)$, $V(x, r):=\mu(B(x, r))=: V_{r}(x)$, and $V(x, y):=\mu(B(x, d(x, y)))$. For any $p \in[1, \infty]$, let $p^{\prime}$ denote its conjugate index, namely, $1 / p+1 / p^{\prime}=1$. For any $a, b \in \mathbb{R}$, let $a \wedge b:=\min \{a, b\}$ and $a \vee b:=\max \{a, b\}$. Finally, for any linear integral operator $T$, we keep the notation $T$ for its integral kernel.

\section{Preliminary Notions and Results}

In this section, we mainly state some preliminary notions and results which are needed to the proof of the main results Theorems $2-4$ below. In particular, we investigate some relations among the boundedness of $\left\{\Pi_{j}\right\}_{j=1}^{3}$.

We first recall the notions of some function spaces. Let $q \in(0, \infty]$. The Lebesgue space $L^{q}(\mathcal{X})$ is defined to be the set of all $\mu$-measurable functions $f$ on $\mathcal{X}$ such that, if $q \in(0, \infty)$,

$$
\|f\|_{L^{q}(\mathcal{X})}:=\left[\int_{\mathcal{X}}|f(x)|^{q} d \mu(x)\right]^{1 / q}<\infty
$$


if $q=\infty,\|f\|_{L^{\infty}(\mathcal{X})}:=\operatorname{ess}_{x \in \mathcal{X}}|f(x)|<\infty$, where ess sup $\sup _{x \in \mathcal{X}}|f(x)|$ denotes the essential supremum of $|f|$ on $\mathcal{X}$. Denote by $L_{\text {loc }}^{1}(\mathcal{X})$ the space of all locally integrable functions.

Let $s \in(0,1]$ and denote by $C(\mathcal{X})$ the space of all continuous functions on $\mathcal{X}$. Then the homogeneous and inhomogeneous spaces $C^{s}(\mathcal{X})$ and $\dot{C}^{s}(\mathcal{X})$ of s-Hölder continuous functions on $\mathcal{X}$ are, respectively, defined by setting

$$
C^{s}(\mathcal{X}):=\left\{f \in C(\mathcal{X}):\|f\|_{C^{s}(\mathcal{X})}<\infty\right\} \quad \text { and } \quad \dot{C}^{s}(\mathcal{X}):=\left\{f \in C(\mathcal{X}):\|f\|_{\dot{C}^{s}(\mathcal{X})}<\infty\right\}
$$

with

$$
\|f\|_{C^{s}(\mathcal{X})}:=\|f\|_{L^{\infty}(\mathcal{X})}+\|f\|_{\dot{C}^{s}(\mathcal{X})} \quad \text { and } \quad\|f\|_{\dot{C}^{s}(\mathcal{X})}:=\sup _{\{(x, y) \in \mathcal{X} \times \mathcal{X}: x \neq y\}} \frac{|f(x)-f(y)|}{[d(x, y)]^{s}} .
$$

Moreover, the space $C_{b}^{s}(\mathcal{X})$ of all s-Hölder continuous functions with bounded support on $\mathcal{X}$ is defined by setting

$$
C_{b}^{s}(\mathcal{X}):=\left\{f \in C^{s}(\mathcal{X}): f \text { has bounded support }\right\},
$$

where we equip $C_{b}^{s}(\mathcal{X})$ with the usual strict inductive limit topology (see, for instance, ([36], p. 273) and ([33], p. 23)). A useful subspace $\dot{C}_{b}^{s}(\mathcal{X})$ of $C_{b}^{s}(\mathcal{X})$ is defined by setting $\stackrel{\circ}{S}_{b}^{s}(\mathcal{X}):=$ $\left\{f \in C_{b}^{s}(\mathcal{X}): \int_{\mathcal{X}} f(x) d \mu(x)=0\right\}$. Moreover, the dual space $\left(C_{b}^{s}(\mathcal{X})\right)^{\prime}\left[\operatorname{resp} .,\left(\dot{C}_{b}^{s}(\mathcal{X})\right)^{\prime}\right]$ of $C_{b}^{s}(\mathcal{X})$ [resp., $\left.\dot{C}_{b}^{s}(\mathcal{X})\right]$ is defined to be the set of all linear functionals on $C_{b}^{s}(\mathcal{X})$ [resp., on $\dot{C}_{b}^{s}(\mathcal{X})$ ] equipped with the weak-* topology.

Definition 1 ([27,32,35]). Let $s \in(0,1]$. A function $K:(\mathcal{X} \times \mathcal{X}) \backslash\{(x, x): x \in \mathcal{X}\} \rightarrow \mathbb{C}$ is called an s-Calderón-Zygmund kernel if there exists a positive constant $C_{(K)}$, depending on $K$, such that

(i) for any $x, y \in \mathcal{X}$ with $x \neq y$,

$$
|K(x, y)| \leq C_{(K)} \frac{1}{V(x, y)}
$$

(ii) for any $x, \tilde{x}, y \in \mathcal{X}$ satisfying $d(x, \widetilde{x}) \leq\left(2 A_{0}\right)^{-1} d(x, y)$ with $x \neq y$,

$$
|K(x, y)-K(\widetilde{x}, y)| \leq C_{(K)}\left[\frac{d(x, \widetilde{x})}{d(x, y)}\right]^{s} \frac{1}{V(x, y)}
$$

and

$$
|K(y, x)-K(y, \widetilde{x})| \leq C_{(K)}\left[\frac{d(x, \widetilde{x})}{d(x, y)}\right]^{s} \frac{1}{V(x, y)} .
$$

A linear operator $T: C_{b}^{s}(\mathcal{X}) \rightarrow\left(C_{b}^{s}(\mathcal{X})\right)^{\prime}$ is called an s-Calderón-Zygmund operator if $T$ can be extended to a bounded linear operator on $L^{2}(\mathcal{X})$ and if there exists an s-Calderón-Zygmund kernel $K$ such that, for any $f \in C_{b}^{s}(\mathcal{X})$ and $x \notin \operatorname{supp} f, T f(x):=\int_{\mathcal{X}} K(x, y) f(y) d \mu(y)$.

Definition 2 ([28]). Let $p \in(0,1]$ and $q \in[1, \infty] \cap(p, \infty]$. A function $a$ on $\mathcal{X}$ is called a $(p, q)$-atom supported on a ball $B$ if (i) supp $a \subset B$; (ii) $\|a\|_{L^{q}(\mathcal{X})} \leq[\mu(B)]^{1 / q-1 / p}$; (iii) $\int_{\mathcal{X}} a(x) d \mu(x)=0$, here and thereafter, for any measurable function $f$, supp $f:=\{x \in \mathcal{X}$ : $f(x) \neq 0\}$.

A function $f \in\left(\operatorname{Lip}_{1 / p-1}(\mathcal{X})\right)^{\prime}$ when $p \in(0,1)$, or $f \in L^{1}(\mathcal{X})$ when $p=1$, is said to belong to the atomic Hardy space $H_{\mathrm{at}}^{p, q}(\mathcal{X})$ if there exist $(p, q)$-atoms $\left\{a_{j}\right\}_{j=1}^{\infty}$ and numbers 
$\left\{\lambda_{j}\right\}_{j=1}^{\infty} \subset \mathbb{C}$ such that $\sum_{j=1}^{\infty}\left|\lambda_{j}\right|^{p}<\infty$ and $f=\sum_{j=1}^{\infty} \lambda_{j} a_{j}$ in $\left(\operatorname{Lip}_{1 / p-1}(\mathcal{X})\right)^{\prime}$ when $p \in(0,1)$, or in $L^{1}(\mathcal{X})$ when $p=1$. Moreover, the quasi-norm of $f$ in $H_{\mathrm{at}}^{p, q}(\mathcal{X})$ is defined by setting

$$
\|f\|_{H_{\mathrm{at}}^{p, q}(\mathcal{X})}:=\inf \left\{\left[\sum_{j=1}^{\infty}\left|\lambda_{j}\right|^{p}\right]^{1 / p}\right\},
$$

where the infimum is taken over all decompositions of $f$ as above.

Let $p \in(0,1]$. It was shown in ([28], Theorem A) that $H_{\mathrm{at}}^{p, q}(\mathcal{X})$ is independent of the choice of $q \in[1, \infty] \cap(p, \infty]$ and hence simply denoted by $H_{\mathrm{at}}^{p}(\mathcal{X})$.

Definition 3 (([34], Definition 2.2) and ([33], Definition 2.8)). Let $x_{1} \in \mathcal{X}$ be fixed, $r, \vartheta \in$ $(0, \infty)$ and $\kappa \in(0,1]$. The space $\mathcal{G}\left(x_{1}, r, \kappa, \vartheta\right)$ of test functions is defined to be the set of all measurable functions $f$ on $\mathcal{X}$ such that there exists a positive constant $C$ such that

(T1) for any $x \in \mathcal{X}$,

$$
|f(x)| \leq C \frac{1}{\mu\left(B\left(x_{1}, r\right)\right)+V\left(x_{1}, x\right)}\left[\frac{r}{r+d\left(x_{1}, x\right)}\right]^{\vartheta}
$$

(T2) for any $x, y \in \mathcal{X}$ with $d(x, y) \leq\left[r+d\left(x_{1}, x\right)\right] /\left(2 A_{0}\right)$,

$$
|f(x)-f(y)| \leq C\left[\frac{d(x, y)}{r+d\left(x_{1}, x\right)}\right]^{\kappa} \frac{1}{\mu\left(B\left(x_{1}, r\right)\right)+V\left(x_{1}, x\right)}\left[\frac{r}{r+d\left(x_{1}, x\right)}\right]^{\vartheta} .
$$

Moreover, the norm of $f$ in $\mathcal{G}\left(x_{1}, r, \kappa, \vartheta\right)$ is defined by setting

$$
\|f\|_{\mathcal{G}\left(x_{1}, r, \kappa, \vartheta\right)}:=\inf \{C: C \text { satisfies (T1) and (T2) }\} .
$$

It was shown in ([33], pp. 18-20) that, for any $x \in \mathcal{X}$ and $r \in(0, \infty)$,

$$
\mathcal{G}(x, r, \kappa, \vartheta)=\mathcal{G}\left(x_{1}, 1, \kappa, \vartheta\right)
$$

with equivalent norms, but the positive equivalence constants may depend on $x$ and $r$ and that $\mathcal{G}\left(x_{1}, 1, \kappa, \vartheta\right)$ is a Banach space. In what follows, for short, we write $\mathcal{G}(\kappa, \vartheta):=\mathcal{G}\left(x_{1}, 1, \kappa, \vartheta\right)$ and let $\mathcal{G}(\kappa, \vartheta):=\left\{f \in \mathcal{G}(\kappa, \vartheta): \int_{\mathcal{X}} f(x) d \mu(x)=0\right\}$.

Let $\varepsilon \in(0,1], \kappa, \vartheta \in(0, \varepsilon]$ and $\mathcal{G}_{0}^{\varepsilon}(\kappa, \vartheta)$ [resp., $\dot{\mathcal{G}}_{0}^{\varepsilon}(\kappa, \vartheta)$ ] be the completion of the space $\mathcal{G}(\varepsilon, \varepsilon)$ [resp., $\mathcal{G}(\varepsilon, \varepsilon)]$ in the $\mathcal{G}(\kappa, \vartheta)$ norm. Moreover, if $f \in \mathcal{G}_{0}^{\varepsilon}(\kappa, \vartheta)$, we then let

$$
\|f\|_{\mathcal{G}_{0}^{\varepsilon}(\kappa, \vartheta)}:=\|f\|_{\mathcal{G}(\kappa, \vartheta)} .
$$

The dual space $\left(\mathcal{G}_{0}^{\varepsilon}(\kappa, \vartheta)\right)^{\prime}$ [resp., $\left.\left(\mathcal{G}_{0}^{\varepsilon}(\kappa, \vartheta)\right)^{\prime}\right]$ is defined to be the set of all continuous linear functionals on $\mathcal{G}_{0}^{\varepsilon}(\kappa, \vartheta)$ [resp., $\mathcal{G}_{0}^{\varepsilon}(\kappa, \vartheta)$ ] and equipped with the weak-* topology.

We then recall the following system of dyadic cubes given in ([64], Theorem 2.2), which was formulated in ([53], Lemma 2.3).

Lemma 1. Fix constants $c_{0}, C_{0}$ and $\delta$ such that $0<c_{0} \leq C_{0}<\infty, \delta \in(0,1)$, and $12 A_{0}^{3} C_{0} \delta \leq c_{0}$. Assume that a set of points, $\left\{z_{\alpha}^{k}: k \in \mathbb{Z}, \alpha \in \mathscr{A}_{k}\right\} \subset \mathcal{X}$ with

$$
\mathscr{A}_{k} \text { being a countable set of indices for any } k \in \mathbb{Z},
$$

satisfies the following properties: for any $k \in \mathbb{Z}$, (i) $d\left(z_{\alpha}^{k}, z_{\beta}^{k}\right) \geq c_{0} \delta^{k}$ when $\alpha \neq \beta$; (ii) for any $x \in \mathcal{X}, \min _{\alpha \in \mathscr{A}_{k}} d\left(x, z_{\alpha}^{k}\right) \leq C_{0} \delta^{k}$. 
Then there exists a family of sets, $\left\{Q_{\alpha}^{k}: k \in \mathbb{Z}, \alpha \in \mathscr{A}_{k}\right\}$, which is called the system of half-open dyadic cubes, satisfying

(iii) $\mathcal{X}=\bigcup_{\alpha \in \mathscr{A}_{k}} Q_{\alpha}^{k}$ with $\left\{Q_{\alpha}^{k}: \alpha \in \mathscr{A}_{k}\right\}$ mutually disjoint;

(iv) if $\ell \geq k, \alpha \in \mathscr{A}_{k}$ and $\beta \in \mathscr{A}_{\ell}$, then either $Q_{\beta}^{\ell} \subset Q_{\alpha}^{k}$ or $Q_{\alpha}^{k} \cap Q_{\beta}^{\ell}=\varnothing$ holds true;

(v) for any $\alpha \in \mathscr{A}_{k}, B\left(z_{\alpha}^{k}, c_{\natural} \delta^{k}\right) \subset Q_{\alpha}^{k} \subset B\left(z_{\alpha}^{k}, C^{\natural} \delta^{k}\right)$ with $c_{\natural}:=\left(3 A_{0}^{2}\right)^{-1} c_{0}, C^{\natural}:=2 A_{0} C_{0}$ and $z_{\alpha}^{k}$ being called the "center" of $Q_{\alpha}^{k}$.

In what follows, for any $k \in \mathbb{Z}$, let

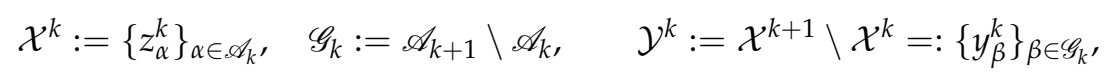

and, for any $y \in \mathcal{X}$, let $d\left(y, \mathcal{Y}^{k}\right):=\inf _{z \in \mathcal{Y}^{k}} d(y, z)$.

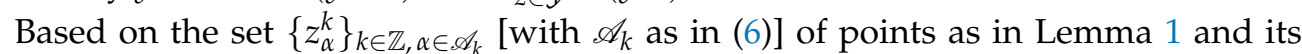
related dyadic cubes, Auscher and Hytönen ([32], Theorem 7.1) constructed the following notable system $\left\{\psi_{\beta}^{k}\right\}_{k \in \mathbb{Z}, \beta \in \mathscr{G}_{k}}$ of regular wavelets on $\mathcal{X}$, which is an orthonormal basis of $L^{2}(\mathcal{X})$.

Lemma 2. There exist constants $C, v \in(0, \infty), a \in(0,1], \eta \in(0,1)$, and regular wavelets

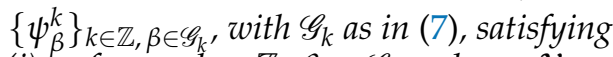

(i) for any $k \in \mathbb{Z}, \beta \in \mathscr{G}_{k}$ and $x \in \mathcal{X}$,

$$
\left|\psi_{\beta}^{k}(x)\right| \leq C \frac{1}{\sqrt{V_{\delta^{k}}\left(y_{\beta}^{k}\right)}} \exp \left\{-v\left[\frac{d\left(y_{\beta^{\prime}}^{k} x\right)}{\delta^{k}}\right]^{a}\right\} ;
$$

(ii) for any $k \in \mathbb{Z}, \beta \in \mathscr{G}_{k}$ and $x, y \in \mathcal{X}$ with $d(x, y) \leq \delta^{k}$,

$$
\left|\psi_{\beta}^{k}(x)-\psi_{\beta}^{k}(y)\right| \leq C\left[\frac{d(x, y)}{\delta^{k}}\right]^{\eta} \frac{1}{\sqrt{V_{\delta^{k}}\left(y_{\beta}^{k}\right)}} \exp \left\{-v\left[\frac{d\left(y_{\beta^{\prime}}^{k} x\right)}{\delta^{k}}\right]^{a}\right\} ;
$$

(iii) for any $k \in \mathbb{Z}$ and $\beta \in \mathscr{G}_{k}, \int_{\mathcal{X}} \psi_{\beta}^{k}(x) d \mu(x)=0$ with $\left\{y_{\beta}^{k}\right\}_{k \in \mathbb{Z}, \beta \in \mathscr{G}_{k}}$ as in (7).

Moreover, the system of regular wavelets $\left\{\psi_{\beta}^{k}\right\}_{k \in \mathbb{Z}, \beta \in \mathscr{G}_{k}}$ is both an orthonormal basis of $L^{2}(\mathcal{X})$ and an unconditional basis of $L^{p}(\mathcal{X})$ for any given $p \in(1, \infty)$.

Definition 4 (([54], Definition 2.7), ([53], Definition 2.4) and ([30], Definition 2.3)). A sequence $\left\{Q_{k}\right\}_{k \in \mathbb{Z}}$ of bounded linear integral operators on $L^{2}(\mathcal{X})$ is called an approximation of the identity with exponential decay (for short, exp-ATI) if there exist constants $C, v \in(0, \infty)$, $a \in(0,1]$ and $\eta \in(0,1)$ such that, for any $k \in \mathbb{Z}$, the kernel of the operator $Q_{k}$, which is still denoted by $Q_{k}$, satisfies

(i) (the identity condition) $\sum_{k=-\infty}^{\infty} Q_{k}=\operatorname{I}$ in $L^{2}(\mathcal{X})$, where I denotes the identity operator on $L^{2}(\mathcal{X})$;

(ii) (the size condition) for any $x, y \in \mathcal{X}$,

$$
\left|Q_{k}(x, y)\right| \leq C R_{k}(x, y)
$$

with

$$
\begin{aligned}
\mathrm{R}_{k}(x, y):= & \frac{1}{\sqrt{V_{\delta^{k}}(x) V_{\delta^{k}}(y)}} \exp \left\{-v\left[\frac{d(x, y)}{\delta^{k}}\right]^{a}\right\} \\
& \times \exp \left\{-v\left[\frac{\max \left\{d\left(x, \mathcal{Y}^{k}\right), d\left(y, \mathcal{Y}^{k}\right)\right\}}{\delta^{k}}\right]^{a}\right\} ;
\end{aligned}
$$


(iii) (the regularity condition) for any $x, \widetilde{x}, y \in \mathcal{X}$ with $d(x, \widetilde{x}) \leq \delta^{k}$,

$$
\left|Q_{k}(x, y)-Q_{k}(\widetilde{x}, y)\right|+\left|Q_{k}(y, x)-Q_{k}(y, \widetilde{x})\right| \leq C\left[\frac{d(x, \widetilde{x})}{\delta^{k}}\right]^{\eta} \mathrm{R}_{k}(x, y) ;
$$

(iv) (the second difference regularity condition) for any $x, \widetilde{x}, y, \widetilde{y} \in \mathcal{X}$ with $d(x, \widetilde{x}) \leq \delta^{k}$ and $d(y, \widetilde{y}) \leq \delta^{k}$,

$$
\left|\left[Q_{k}(x, y)-Q_{k}(\widetilde{x}, y)\right]-\left[Q_{k}(x, \widetilde{y})-Q_{k}(\widetilde{x}, \widetilde{y})\right]\right| \leq C\left[\frac{d(x, \widetilde{x})}{\delta^{k}}\right]^{\eta}\left[\frac{d(y, \widetilde{y})}{\delta^{k}}\right]^{\eta} \mathrm{R}_{k}(x, y) ;
$$

(v) (the cancellation condition) for any $x, y \in \mathcal{X}, \int_{\mathcal{X}} Q_{k}(x, y) d \mu(y)=0=\int_{\mathcal{X}} Q_{k}(x, y) d \mu(x)$.

Remark 1. Let $\left\{\psi_{\beta}^{k}\right\}_{k \in \mathbb{Z}, \beta \in \mathscr{G}_{k}}$ be as in Lemma 2. For any $k \in \mathbb{Z}$ and $x, y \in \mathcal{X}$, let

$$
D_{k}(x, y):=\sum_{\beta \in \mathscr{G}_{k}} \psi_{\beta}^{k}(x) \psi_{\beta}^{k}(y)
$$

It was shown in ([54], $p$. 291) that the sequence $\left\{D_{k}\right\}_{k \in \mathbb{Z}}$ of linear integral operators associated with kernels $\left\{D_{k}(\cdot, \cdot)\right\}_{k \in \mathbb{Z}}$ satisfies all conditions (i)-(v) of Definition 4.

Definition 5 ([53], Definition 2.8). A sequence $\left\{P_{k}\right\}_{k \in \mathbb{Z}}$ of bounded linear integral operators on $L^{2}(\mathcal{X})$ is called an approximation of the identity with exponential decay and integration 1 (for short, 1-exp-ATI) if $\left\{P_{k}\right\}_{k \in \mathbb{Z}}$ has the following properties:

(i) for any $k \in \mathbb{Z}, P_{k}$ satisfies (ii), (iii), and (iv) of Definition 4, but without the exponential decay factor

$$
\exp \left\{-v\left[\frac{\max \left\{d\left(x, \mathcal{Y}^{k}\right), d\left(y, \mathcal{Y}^{k}\right)\right\}}{\delta^{k}}\right]^{a}\right\}
$$

with $\mathcal{Y}^{k}$ as in (7);

(ii) $\int_{\mathcal{X}} P_{k}(x, y) d \mu(y)=1=\int_{\mathcal{X}} P_{k}(y, x) d \mu(y)$ for any $k \in \mathbb{Z}$ and $x \in \mathcal{X}$;

(iii) Let $Q_{k}:=P_{k}-P_{k-1}$ for any $k \in \mathbb{Z}$. Then $\left\{Q_{k}\right\}_{k \in \mathbb{Z}}$ is an exp-ATI.

\section{Remark 2.}

(i) The existence of the 1-exp-ATI is ensured by ([32], Lemma 10.1) (see also ([53], Remark 2.9)).

(ii) For any given $p \in[1, \infty], P_{k}$ and hence $Q_{k}$ are bounded on $L^{p}(\mathcal{X})$ uniformly in $k \in \mathbb{Z}$; see, for instance, ([54], Proposition 2.2(iii)).

(iii) It was shown that $\lim _{k \rightarrow \infty} P_{k}=I$ on $L^{2}(\mathcal{X})$; see, for example, ([53], Remark 2.9).

Definition 6 (([53], Section 3 and Theorem 5.10) and ([62], Definition 1.1)). Let $\kappa, \vartheta \in(0, \eta)$ with $\eta$ as in Lemma 2, $\left\{P_{k}\right\}_{k \in \mathbb{Z}}$ be a 1-exp-ATI and $Q_{k}:=P_{k}-P_{k-1}$ for any $k \in \mathbb{Z}$. Then, for any $f \in\left(\mathcal{G}_{0}^{\eta}(\kappa, \vartheta)\right)^{\prime}$, the non-tangential maximal function $\mathcal{M}_{\rho}(f)$ of $f$, with aperture $\rho \in(0, \infty)$, is defined by setting, for any $x \in \mathcal{X}$,

$$
\mathcal{M}_{\rho}(f)(x):=\sup _{k \in \mathbb{Z}} \sup _{y \in B\left(x, \rho \delta^{k}\right)}\left|P_{k} f(y)\right| .
$$

Moreover, for any $f \in\left(\mathcal{G}_{0}^{\eta}(\kappa, \vartheta)\right)^{\prime}$, the Littlewood-Paley $g$-function $g(f)$ of $f$ is defined by setting, for any $x \in \mathcal{X}$,

$$
g(f)(x):=\left[\sum_{k \in \mathbb{Z}}\left|Q_{k} f(x)\right|^{2}\right]^{1 / 2}
$$


Let $p \in(0, \infty]$ and $\rho \in(0, \infty)$. Then the Hardy spaces $H_{\rho}^{p}(\mathcal{X})$ and $H^{p}(\mathcal{X})$ are defined, respectively, by setting

$$
H_{\rho}^{p}(\mathcal{X}):=\left\{f \in\left(\mathcal{G}_{0}^{\eta}(\kappa, \vartheta)\right)^{\prime}:\|f\|_{H_{\rho}^{p}(\mathcal{X})}:=\left\|\mathcal{M}_{\rho}(f)\right\|_{L^{p}(\mathcal{X})}<\infty\right\}
$$

and

$$
H^{p}(\mathcal{X}):=\left\{f \in\left(\dot{\mathcal{G}}_{0}^{\eta}(\kappa, \vartheta)\right)^{\prime}:\|f\|_{H^{p}(\mathcal{X})}:=\|g(f)\|_{L^{p}(\mathcal{X})}<\infty\right\} .
$$

Remark 3. Let $\rho \in(0, \infty), p \in(n /(n+\eta), 1]$ and $\kappa, \vartheta \in(n(1 / p-1)$, $\eta)$. It was shown in ([62], Remark 1.2) and ([60], Theorem 6.1) that

(i) $H_{\rho}^{p}(\mathcal{X})$ and $H_{\mathrm{at}}^{p}(\mathcal{X})$ coincide with equivalent quasi-norms;

(ii) $H^{p}(\mathcal{X})=H_{\mathrm{at}}^{p}(\mathcal{X})$ with equivalent quasi-norms as subspaces of $\left(\mathscr{\mathcal { G }}_{0}^{\eta}(\kappa, \vartheta)\right)^{\prime}$;

(ii) for any given $p \in(1, \infty), H_{\rho}^{p}(\mathcal{X})=L^{p}(\mathcal{X})=H^{p}(\mathcal{X})$ with equivalent norms.

We now introduce the following notion of paraproducts on $\mathcal{X}$ adapted from ([48], (3.2)).

Definition 7. Let $\kappa, \vartheta \in(0, \eta)$ with $\eta$ as in Lemma 2. Let $\left\{P_{j}\right\}_{j \in \mathbb{Z}}$ be a 1-exp-ATI and $Q_{j}:=$ $P_{j}-P_{j-1}$ for any $j \in \mathbb{Z}$. Then the paraproduct $\Pi_{3}$ is formally defined by setting, for any $f \in\left(\mathcal{G}_{0}^{\eta}(\kappa, \vartheta)\right)^{\prime}, g \in\left(\mathcal{G}_{0}^{\eta}(\kappa, \vartheta)\right)^{\prime}$ and $x \in \mathcal{X}$,

$$
\Pi_{3}(f, g)(x):=\sum_{j \in \mathbb{Z}} Q_{j}(f)(x) Q_{j}(g)(x),
$$

where the series converges in $\left(\mathcal{G}_{0}^{\eta}(\kappa, \vartheta)\right)^{\prime}$.

Remark 4. In Theorems 2 and 3 below, we prove that $\Pi_{3}(f, g)$ in Definition 7 is well defined for any $(f, g) \in H^{p}(\mathcal{X}) \times H^{q}(\mathcal{X})$ with $p, q \in(0, \infty)$ and any $(f, g) \in \operatorname{BMO}(\mathcal{X}) \times C_{b}^{\eta}(\mathcal{X})$.

Definition 8. Let $\kappa, \vartheta \in(0, \eta)$ with $\eta$ be as in Lemma 2. Let $\left\{P_{j}\right\}_{j \in \mathbb{Z}}$ be a 1-exp-ATI and $Q_{j}:=P_{j}-P_{j-1}$ for any $j \in \mathbb{Z}$. Then the paraproducts $\Pi_{1}$ and $\Pi_{2}$ are formally defined, respectively, by setting

(i) for any $f \in\left(\mathcal{G}_{0}^{\eta}(\kappa, \vartheta)\right)^{\prime}, g \in\left(\mathcal{G}_{0}^{\eta}(\kappa, \vartheta)\right)^{\prime}$ and $x \in \mathcal{X}$,

$$
\Pi_{1}(f, g)(x):=\sum_{j \in \mathbb{Z}} Q_{j}(f)(x) P_{j}(g)(x) ;
$$

(ii) for any $f \in\left(\mathcal{G}_{0}^{\eta}(\kappa, \vartheta)\right)^{\prime}, g \in\left(\mathcal{G}_{0}^{\eta}(\kappa, \vartheta)\right)^{\prime}$ and $x \in \mathcal{X}$,

$$
\Pi_{2}(f, g)(x):=\sum_{j \in \mathbb{Z}} P_{j}(f)(x) Q_{j}(g)(x),
$$

where the above two series converge in $\left(\mathcal{G}_{0}^{\eta}(\kappa, \vartheta)\right)^{\prime}$.

\section{Remark 5.}

(i) In Theorem 4 below, we show that $\Pi_{1}(f, g)$ in Definition 8 is well defined for any $(f, g) \in$ $L^{\infty}(\mathcal{X}) \times C_{b}^{\eta}(\mathcal{X})$

(ii) Due to the fact that $\Pi_{2}(f, g)=\Pi_{1}(g, f)$ for any proper functions $f$ and $g$, we conclude that $\Pi_{2}$ shares corresponding boundedness to $\Pi_{1}$ as in Theorem 4 below.

To prove Theorem 3 below, we need to recall the $T(1)$ theorem from ([32], Section 12). Let $\sigma \in(0,1)$ and $s \in(0, \sigma]$. A linear continuous operator $T: C_{b}^{s}(\mathcal{X}) \rightarrow\left(C_{b}^{s}(\mathcal{X})\right)^{\prime}$ is 
said to have weak boundedness property $\operatorname{WBP}(\sigma)$ if there exists a positive constant $C_{1}$ such that, for any $f, g \in C_{b}^{\sigma}(\mathcal{X})$ normalized by $\|f\|_{L^{\infty}(\mathcal{X})}+r^{\sigma}\|f\|_{\dot{C}^{\sigma}(\mathcal{X})} \leq 1$ and $\|g\|_{L^{\infty}(\mathcal{X})}+$ $r^{\sigma}\|g\|_{\dot{C}^{\sigma}(\mathcal{X})} \leq 1$, with support in some ball $B(x, r)(x \in \mathcal{X}$ and $r \in(0, \infty))$,

$$
|(T f, g)| \leq C_{1} V(x, r) .
$$

As for $T(1)$ with $T$ associated with the $s$-Calderón-Zygmund kernel, it is defined as a continuous linear functional on $\stackrel{C}{b}_{b}^{s}(\mathcal{X})$ by setting

$$
\langle T(1), f\rangle:=\langle T(g), f\rangle+\int_{\mathcal{X}}(1-g(x)) T^{*}(f)(x) d \mu(x),
$$

where $g: \mathcal{X} \rightarrow \mathbb{R}$ satisfies that there exists a ball $B\left(x_{0}, r\right) \supset$ supp $f$ such that, for any $x \in \mathcal{X}, \mathbf{1}_{B\left(x_{0}, r\right)}(x) \leq g(x) \leq \mathbf{1}_{B\left(x_{0}, 2 A_{0} r\right)}(x)$. It is not difficult to show that both of the two terms in the right hand side of (8) are well defined.

Lemma 3. Let $\sigma \in(0,1), s \in(0, \sigma],(\mathcal{X}, d, \mu)$ be any space of homogeneous type and $T$ be associated to an s-Calderón-Zygmund kernel. Then $T$ can be extended to a bounded operator on $L^{2}(\mathcal{X})$ if and only if $T$ has $\operatorname{WBP}(s)$ and $T(1), T^{*}(1) \in \operatorname{BMO}(\mathcal{X})$.

At the end of this section, we use the Abel summation formula to make some links among the boundedness of $\Pi_{1}, \Pi_{2}$ and $\Pi_{3}$ in some sense, which plays an important role in the proof of Theorem 4 below. In what follows, for any $N \in \mathbb{Z}$ and suitable functions $f$ and $g$,

$$
\Pi_{1}^{(N)}(f, g):=\sum_{j=-N}^{N} P_{j}(f) Q_{j}(g), \quad \Pi_{2}^{(N)}(f, g):=\sum_{j=-N}^{N} Q_{j}(f) P_{j}(g)
$$

and $\Pi_{3}^{(N)}(f, g):=\sum_{j=-N}^{N} Q_{j}(f) Q_{j}(g)$.

Theorem 1. Assume that there exists a positive constant $C$ such that, for any $N \in \mathbb{N}, f \in L^{2}(\mathcal{X})$ and $g \in L^{\infty}(\mathcal{X})$,

$$
\left\|\Pi_{2}^{(N)}(f, g)\right\|_{L^{2}(\mathcal{X})}+\left\|\Pi_{3}^{(N)}(f, g)\right\|_{L^{2}(\mathcal{X})} \leq C\|f\|_{L^{2}(\mathcal{X})}\|g\|_{L^{\infty}(\mathcal{X})} .
$$

Then $\Pi_{1}$ defined as in Definition 8 is bounded from $L^{2}(\mathcal{X}) \times L^{\infty}(\mathcal{X})$ into $L^{2}(\mathcal{X})$.

Proof. Let $f \in L^{2}(\mathcal{X})$ and $g \in L^{\infty}(\mathcal{X})$. For any $N \in \mathbb{N}$, by the Abel summation formula, we know that

$$
\begin{aligned}
\Pi_{1}^{(N)}(f, g)= & \sum_{j=-N}^{N} P_{j}(f) Q_{j}(g)=\sum_{j=-N}^{N} P_{j}(f)\left[P_{j}(g)-P_{j-1}(g)\right] \\
= & \sum_{j=-N}^{N} P_{j}(f) P_{j}(g)-\sum_{j=-N}^{N} P_{j}(f) P_{j-1}(g) \\
= & \sum_{j=-N}^{N} P_{j}(f) P_{j}(g)-\sum_{j=-N-1}^{N-1} P_{j+1}(f) P_{j}(g) \\
= & P_{N+1}(f) P_{N}(g)-P_{-N}(f) P_{-N-1}(g) \\
& +\sum_{j=-N}^{N}\left[P_{j}(f)-P_{j+1}(f)\right] P_{j}(g) \\
= & P_{N+1}(f) P_{N}(g)-P_{-N}(f) P_{-N-1}(g)
\end{aligned}
$$




$$
\begin{aligned}
& -\sum_{j=-N}^{N} Q_{j+1}(f) P_{j}(g) \\
= & P_{N+1}(f) P_{N}(g)-P_{-N}(f) P_{-N-1}(g) \\
& -\sum_{j=-N}^{N} Q_{j+1}(f) P_{j+1}(g)+\sum_{j=-N}^{N} Q_{j+1}(f)\left[P_{j+1}(g)-P_{j}(g)\right] \\
= & P_{N+1}(f) P_{N}(g)-P_{-N}(f) P_{-N-1}(g) \\
& -\sum_{j=-N}^{N} Q_{j+1}(f) P_{j+1}(g)+\sum_{j=-N}^{N} Q_{j+1}(f) Q_{j+1}(g) \\
= & P_{N+1}(f) P_{N}(g)-P_{-N}(f) P_{-N-1}(g) \\
& -\sum_{j=-N+1}^{N+1} Q_{j}(f) P_{j}(g)+\sum_{j=-N+1}^{N+1} Q_{j}(f) Q_{j}(g) \\
= & P_{N+1}(f) P_{N}(g)-P_{-N}(f) P_{-N-1}(g)+Q_{-N}(f) P_{-N}(g) \\
& -Q_{N+1}(f) P_{N+1}(g)+Q_{N+1}(f) Q_{N+1}(g)-Q_{-N}(f) Q_{-N}(g) \\
& -\Pi_{2}^{(N)}(f, g)+\Pi_{3}^{(N)}(f, g) .
\end{aligned}
$$

From this, (9) and Remark 2(ii), we deduce that

$$
\begin{aligned}
\left\|\Pi_{1}^{(N)}(f, g)\right\|_{L^{2}(\mathcal{X})} \leq & \left\|P_{N+1}(f) P_{N}(g)\right\|_{L^{2}(\mathcal{X})}+\left\|P_{-N}(f) P_{-N-1}(g)\right\|_{L^{2}(\mathcal{X})} \\
& +\left\|Q_{-N}(f) P_{-N}(g)\right\|_{L^{2}(\mathcal{X})}+\left\|Q_{N+1}(f) P_{N+1}(g)\right\|_{L^{2}(\mathcal{X})} \\
& +\left\|Q_{N+1}(f) Q_{N+1}(g)\right\|_{L^{2}(\mathcal{X})}+\left\|Q_{-N}(f) Q_{-N}(g)\right\|_{L^{2}(\mathcal{X})} \\
& +\left\|\Pi_{2}^{(N)}(f, g)\right\|_{L^{2}(\mathcal{X})}+\left\|\Pi_{3}^{(N)}(f, g)\right\|_{L^{2}(\mathcal{X})} \\
\lesssim & \|f\|_{L^{2}(\mathcal{X})}\|g\|_{L^{\infty}(\mathcal{X})}
\end{aligned}
$$

which, combined with the Fatou lemma, implies that

$$
\left\|\Pi_{1}(f, g)\right\|_{L^{2}(\mathcal{X})} \leq \limsup _{N \rightarrow \infty}\left\|\Pi_{1}^{(N)}(f, g)\right\|_{L^{2}(\mathcal{X})} \lesssim\|f\|_{L^{2}(\mathcal{X})}\|g\|_{L^{\infty}(\mathcal{X})} .
$$

This completes the proof of Theorem 1.

\section{Boundedness of Paraproducts $\left\{\Pi_{j}\right\}_{j=1}^{3}$}

This section is devoted to the proofs of the main results of this article on the boundedness of paraproducts $\left\{\Pi_{j}\right\}_{j=1}^{3}$.

We now state the first main result of this article as follows.

Theorem 2. Let $\eta$ be as in Lemma $2, p, q, r \in(n /(n+\eta), \infty)$ with $1 / r=1 / p+1 / q$, and $\kappa, \vartheta \in(\max \{0, n(1 / r-1)\}, \eta)$. Then the paraproduct $\Pi_{3}$ as in Definition 7 is a bounded bilinear operator from $H^{p}(\mathcal{X}) \times H^{q}(\mathcal{X})$ into $L^{r}(\mathcal{X})$.

\section{Remark 6.}

(i) Theorem 2 is an extension of ([48], Lemma 3.3).

(ii) It is still unclear whether $\Pi_{1}$ and $\Pi_{2}$ can be extended to bounded operators from $H^{p}(\mathcal{X}) \times$ $H^{q}(\mathcal{X})$ into $H^{r}(\mathcal{X})$ or not.

The following result is an easy consequence of Theorem 2, we omit the details here.

Corollary 1. Let $q \in(1, \infty)$ and $q^{\prime}:=q /(q-1)$. Then the paraproduct $\Pi_{3}$ as in Definition 7 is a bounded bilinear operator from $L^{q}(\mathcal{X}) \times L^{q^{\prime}}(\mathcal{X})$ into $L^{1}(\mathcal{X})$. 
Now, we are ready to prove Theorem 2 .

Proof of Theorem 2. Let $p, q, r, \eta, \kappa, \vartheta$, and $\Pi_{3}$ be as in Theorem 2. For any $(f, g) \in$ $H^{p}(\mathcal{X}) \times H^{q}(\mathcal{X})$, we know that $f, g \in\left(\mathcal{G}_{0}^{\eta}(\kappa, \vartheta)\right)^{\prime}$. By the Hölder inequality, we immediately have

$$
\begin{aligned}
\left\|\Pi_{3}(f, g)\right\|_{L^{r}(\mathcal{X})} & \leq\left\|\sum_{j \in \mathbb{Z}}\left|Q_{j}(f) Q_{j}(g)\right|\right\|_{L^{r}(\mathcal{X})} \\
& \leq\left\|\left[\sum_{j \in \mathbb{Z}}\left|Q_{j}(f)\right|^{2}\right]^{1 / 2}\left[\sum_{j \in \mathbb{Z}}\left|Q_{j}(g)\right|^{2}\right]^{1 / 2}\right\| \\
& \leq\left\|\left[\sum_{j^{\prime} \in \mathbb{Z}}\left|Q_{j}(f)\right|^{2}\right]^{1 / 2}\right\|\left\|_{L^{p}(\mathcal{X})}\right\|\left[\sum_{j \in \mathbb{Z}}\left|Q_{j}(g)\right|^{2}\right]^{1 / 2} \|_{L^{q}(\mathcal{X})} \\
& =\|f\|_{H^{p}(\mathcal{X})}\|g\|_{H^{q}(\mathcal{X})},
\end{aligned}
$$

which completes the proof of Theorem 2.

Then we state other two main results of this article, which give various endpoint estimates of $\Pi_{3}$ and $\Pi_{1}$. In what follows, the weak Lebesgue space $L^{1, \infty}(\mathcal{X})$ is defined to be the set of all $\mu$-measurable functions $f$ on $\mathcal{X}$ such that

$$
\|f\|_{L^{1, \infty}(\mathcal{X})}:=\sup _{\lambda \in(0, \infty)}[\lambda \mu(\{x \in \mathcal{X}:|f(x)|>\lambda\})]<\infty,
$$

and the space $\operatorname{BMO}(\mathcal{X})$ the set of all locally integrable functions $f$ on $\mathcal{X}$ such that

$$
\|f\|_{\mathrm{BMO}(\mathcal{X})}:=\sup _{B \subset \mathcal{X}} \frac{1}{\mu(B)} \int_{B}\left|f(x)-m_{B}(f)\right| d \mu(x)<\infty,
$$

where the supremum is taken over all balls of $\mathcal{X}$ and, here and thereafter, for any locally integrable function $f$ and a ball $B \subset \mathcal{X}, m_{B}(f):=\frac{1}{\mu(B)} \int_{B} f(y) d \mu(y)$.

Theorem 3. Let $\eta$ be as in Lemma $2, q \in(1, \infty), \kappa, \vartheta \in(\max \{0, n(1 / q-1)\}, \eta)$, and $\Pi_{3}$ be as in Definition 7. Assume that the exp-ATI, $\left\{Q_{j}\right\}_{j \in \mathbb{Z}}$, further satisfies

(a) $Q_{j}^{*}=Q_{j}$ and $Q_{j}^{2}=Q_{j}$ on $L^{2}(\mathcal{X})$ for any $j \in \mathbb{Z}$, namely, $\left\{Q_{j}\right\}_{j \in \mathbb{Z}}$ are projection operators on $L^{2}(\mathcal{X})$.

(b) $\quad \sum_{j \in \mathbb{Z}} Q_{j}=I$ in $H_{\mathrm{at}}^{1}(\mathcal{X})$.

Then $\Pi_{3}$ can be extended to a bounded bilinear operator

(i) from $\mathrm{BMO}(\mathcal{X}) \times L^{q}(\mathcal{X})$ into $L^{q}(\mathcal{X})$;

(ii) from $\mathrm{BMO}(\mathcal{X}) \times H_{\mathrm{at}}^{1}(\mathcal{X})$ into $L^{1}(\mathcal{X})$;

(iii) from $\operatorname{BMO}(\mathcal{X}) \times L^{\infty}(\mathcal{X})$ into $\operatorname{BMO}(\mathcal{X})$;

(iv) from $\operatorname{BMO}(\mathcal{X}) \times L^{1}(\mathcal{X})$ into $L^{1, \infty}(\mathcal{X})$;

(v) from $L^{q}(\mathcal{X}) \times L^{\infty}(\mathcal{X})$ into $L^{q}(\mathcal{X})$;

(vi) from $L^{1}(\mathcal{X}) \times L^{\infty}(\mathcal{X})$ into $L^{1, \infty}(\mathcal{X})$.

Remark 7. From ([32], Section 10) and ([65], Theorem 3.10), it follows that the sequence $\left\{D_{k}\right\}_{k \in \mathbb{Z}}$ in Remark 1 still satisfies all the assumptions in Theorem 3. Thus, Theorem 3(ii) is an extension of ([48], Theorem 4.9).

The following result is a variant of ([62], Theorem 7). 
Theorem 4. Let $\eta$ be as in Lemma $2, q \in(1, \infty), p \in\left(\frac{n}{n+\eta}, \infty\right), \kappa, \vartheta \in(\max \{0, n(1 / p-$ 1) $\}, \eta)$, and $\Pi_{1}$ be as in Definition 8. Assume that the exp-ATI, $\left\{Q_{j}\right\}_{j \in \mathbb{Z}}$, further satisfies, for any $f \in L^{\infty}(\mathcal{X})$ and $(1,2)$-atom $h$ supported on some ball $B_{0}$,

$$
\int_{\mathcal{X}} \Pi_{1}(f, h)(x) d \mu(x)=0 .
$$

Then $\Pi_{1}$ can be extended to a bounded bilinear operator

(i) from $L^{\infty}(\mathcal{X}) \times H^{p}(\mathcal{X})$ into $H^{p}(\mathcal{X})$;

(ii) from $L^{\infty}(\mathcal{X}) \times H_{\text {at }}^{1}(\mathcal{X})$ into $L^{1}(\mathcal{X})$;

(iii) from $L^{\infty}(\mathcal{X}) \times L^{\infty}(\mathcal{X})$ into $\mathrm{BMO}(\mathcal{X})$;

(iv) from $L^{\infty}(\mathcal{X}) \times L^{1}(\mathcal{X})$ into $L^{1, \infty}(\mathcal{X})$.

(v) from $L^{q}(\mathcal{X}) \times L^{\infty}(\mathcal{X})$ into $L^{q}(\mathcal{X})$;

(vi) from $L^{1}(\mathcal{X}) \times L^{\infty}(\mathcal{X})$ into $L^{1, \infty}(\mathcal{X})$.

\section{Remark 8.}

(i) Let $f \in L^{\infty}(\mathcal{X}) \subset \operatorname{BMO}(\mathcal{X})$, $h$ be a $(1,2)$-atom and $Q_{k}:=D_{k}(k \in \mathbb{Z})$ be as in Remark 1 . By $\left([48], p\right.$. 985, lines 1-3 from the bottom), we have $\Pi_{1}(f, h)=H+h m_{B_{0}}(f)$ with $H \in$ $H_{\mathrm{at}}^{1}(\mathcal{X})$, which, together with the fact that, for any $G \in H_{\mathrm{at}}^{1}(\mathcal{X}), \int_{\mathcal{X}} G(x) d \mu(x)=0$, further implies that $\int_{\mathcal{X}} \Pi_{1}(f, h)(x) d \mu(x)=0$. Therefore, the sequence $\left\{D_{k}\right\}_{k \in \mathbb{Z}}$ in Remark 1 still satisfies all the assumptions in Theorem 4.

(ii) It is still unknown what happens if we replace $f \in L^{\infty}(\mathcal{X})$ (resp., $g \in L^{\infty}(\mathcal{X})$ ) by $f \in$ $\mathrm{BMO}(\mathcal{X})($ resp., $g \in \mathrm{BMO}(\mathcal{X}))$ in Theorem 4.

As in ([30], Remark 3.3) or ([62], Remark 1.8), the following estimates are important to escape the dependence on the RD-condition (2). For any given $a, c \in(0, \infty)$, and, for any $r \in(0, \infty)$ and $x \in \mathcal{X}$,

$$
\sum_{\left\{k \in \mathbb{Z}: \delta^{k} \geq r\right\}} \frac{1}{V_{\delta^{k}}(x)} \exp \left\{-c\left[\frac{d\left(x, \mathcal{Y}^{k}\right)}{\delta^{k}}\right]^{a}\right\} \lesssim \frac{1}{V_{r}(x)}
$$

(see ([32], Lemma 8.3)) and, for any $x, y \in \mathcal{X}$ with $x \neq y$,

$$
\sum_{k \in \mathbb{Z}} \frac{1}{V_{\delta^{k}}(x)} \exp \left\{-c\left[\frac{d(x, y)}{\delta^{k}}\right]^{a}\right\} \exp \left\{-c\left[\frac{d\left(x, \mathcal{Y}^{k}\right)}{\delta^{k}}\right]^{a}\right\} \lesssim \frac{1}{V(x, y)},
$$

where the implicit positive constant is independent of $x$ and $y$ (see ([54], Lemma 4.9)), which essentially connect the geometrical properties of $\mathcal{X}$ expressed via its equipped quasi-metric $d$, dyadic reference points and dyadic cubes.

Now, we are ready to prove Theorem 3.

Proof of Theorem 3. Without loss of generality, we may assume that the sum $\sum_{j \in \mathbb{Z}}$ in $\Pi_{3}(f, g)$ is a finite sum $\sum_{j=-N}^{N}$ for any fixed $N \in \mathbb{N}$, see ([66], pp. 302-305) for some details.

We first prove (i)-(iv) of this theorem. To this end, we temporarily fix an $f \in \operatorname{BMO}(\mathcal{X})$. For any $x \in \mathcal{X}$, we write

$$
\begin{aligned}
\Pi_{3}(f, g)(x) & =\sum_{j \in \mathbb{Z}} Q_{j}(f)(x) Q_{j}(g)(x)=\int_{\mathcal{X}}\left[\sum_{j \in \mathbb{Z}} Q_{j}(x, y) Q_{j}(f)(x)\right] g(y) d \mu(y) \\
& =: \int_{\mathcal{X}} K_{f}^{(3)}(x, y) g(y) d \mu(y)=: K_{f}^{(3)}(g)(x),
\end{aligned}
$$


where $K_{f}^{(3)}$ is an integral operator associated with the kernel defined by setting, for any $x, y \in \mathcal{X}$,

$$
K_{f}^{(3)}(x, y):=\sum_{j \in \mathbb{Z}} Q_{j}(x, y) Q_{j}(f)(x)
$$

To prove (i)-(iv) of this theorem, the key point is the proof of the boundedness of $K_{f}^{(3)}$ on $L^{2}(\mathcal{X})$, where we need some ideas from ([67], Remark 4.4.5).

We first claim that $K_{f}^{(3)}$ has $\operatorname{WBP}(\eta)$ and hence maps from $C_{b}^{\eta}(\mathcal{X})$ into $\left(C_{b}^{\eta}(\mathcal{X})\right)^{\prime}$. Indeed, let $g, h \in C_{b}^{\eta}(\mathcal{X})$, supported on some ball $B\left(x_{0}, r_{0}\right)$ with $x_{0} \in \mathcal{X}$ and $r_{0} \in(0, \infty)$, be normalized by

$$
\|g\|_{L^{\infty}(\mathcal{X})}+r_{0}^{\eta}\|g\|_{\dot{C}_{b}^{\eta}(\mathcal{X})} \leq 1 \quad \text { and } \quad\|h\|_{L^{\infty}(\mathcal{X})}+r_{0}^{\eta}\|h\|_{\dot{C}_{b}^{\eta}(\mathcal{X})} \leq 1
$$

Then, by the fact from ([62], (2.3)) that

$$
\sup _{j \in \mathbb{Z}}\left\|Q_{j}(f)\right\|_{L^{\infty}(\mathcal{X})} \lesssim\|f\|_{\mathrm{BMO}(\mathcal{X})}
$$

and the Hölder inequality, we conclude that

$$
\begin{aligned}
\left|\left\langle K_{f}^{(3)}(g), h\right\rangle\right| & =\left|\left\langle\Pi_{3}(f, g), h\right\rangle\right|=\left|\int_{\mathcal{X}} \Pi_{3}(f, g)(x) h(x) d \mu(x)\right| \\
& \leq \sum_{j \in \mathbb{Z}} \int_{\mathcal{X}}\left|Q_{j}(f)(x)\right|\left|Q_{j}(g)(x)\right||h(x)| d \mu(x) \\
& \lesssim\|f\|_{\mathrm{BMO}(\mathcal{X})} \sum_{j \in \mathbb{Z}} \int_{\mathcal{X}}\left|Q_{j}(g)(x)\right||h(x)| d \mu(x) \\
& \lesssim\|f\|_{\mathrm{BMO}(\mathcal{X})} \sum_{j \in \mathbb{Z}}\left\|Q_{j}(g)\right\|_{L^{2}(\mathcal{X})}\|h\|_{L^{2}(\mathcal{X})} \\
& \lesssim\|f\|_{\mathrm{BMO}(\mathcal{X})}\left[V\left(x_{0}, r_{0}\right)\right]^{1 / 2} \sum_{j \in \mathbb{Z}}\left\|Q_{j}(g)\right\|_{L^{2}(\mathcal{X})} .
\end{aligned}
$$

Thus, to prove the above claim, it suffices to show that

$$
\sum_{j \in \mathbb{Z}}\left\|Q_{j}(g)\right\|_{L^{2}(\mathcal{X})} \lesssim\left[V\left(x_{0}, r_{0}\right)\right]^{1 / 2} .
$$

We further consider the following two cases.

(Case 1) $\delta^{j} \geq r_{0}$. Choose a fixed $x_{1} \in B\left(x_{0}, 2 r_{0}\right) \backslash B\left(x_{0}, r_{0}\right)$. Then, by (v) and (ii) of Definition 4 and (1), we have

$$
\begin{aligned}
& \left|Q_{j}(g)(x)\right| \\
& \quad=\left|\int_{B\left(x_{0}, r_{0}\right)} Q_{j}(x, y)\left[g(y)-g\left(x_{1}\right)\right] d \mu(y)\right| \\
& \leq \int_{B\left(x_{0}, r_{0}\right)}\left|Q_{j}(x, y) \|\right| g(y)-g\left(x_{1}\right) \mid d \mu(y) \\
& \quad \lesssim \int_{B\left(x_{0}, r_{0}\right)} \frac{1}{V_{\delta^{j}}(x)} \exp \left\{-\frac{v}{2}\left[\frac{d(x, y)}{\delta^{j}}\right]^{a}\right\} \exp \left\{-v\left[\frac{d\left(y, \mathcal{Y}^{j}\right)}{\delta^{j}}\right]^{a}\right\}\left[d\left(y, x_{1}\right)\right]^{\eta}\|g\|_{\dot{C}_{b}^{\eta}(\mathcal{X})} d \mu(y) \\
& \quad \lesssim r_{0}^{\eta}\|g\|_{\dot{C}_{b}^{\eta}(\mathcal{X})} \int_{B\left(x_{0}, r_{0}\right)} \frac{1}{V_{\delta^{j}}(x)} \exp \left\{-\frac{v}{2}\left[\frac{d(x, y)}{\delta^{j}}\right]^{a}\right\} \exp \left\{-\frac{v}{2}\left[\frac{d\left(y, x_{0}\right)}{\delta^{j}}\right]^{a}\right\} \\
& \quad \times \exp \left\{-v\left[\frac{d\left(y, \mathcal{Y}^{j}\right)}{\delta^{j}}\right]^{a}\right\} \exp \left\{-v\left[\frac{d\left(y, x_{0}\right)}{\delta^{j}}\right]^{a}\right\} d \mu(y)
\end{aligned}
$$




$$
\begin{aligned}
& \lesssim \int_{B\left(x_{0}, r_{0}\right)} \frac{1}{V_{\delta^{j}}(x)} \exp \left\{-\frac{v}{2}\left[\frac{d\left(x, x_{0}\right)}{A_{0} \delta^{j}}\right]^{a}\right\} \exp \left\{-v\left[\frac{d\left(x_{0}, \mathcal{Y}^{j}\right)}{A_{0} \delta^{j}}\right]^{a}\right\} d \mu(y) \\
& \lesssim \frac{V\left(x_{0}, r_{0}\right)}{V_{\delta^{j}}\left(x_{0}\right)} \exp \left\{-\frac{v}{4}\left[\frac{d\left(x, x_{0}\right)}{A_{0} \delta^{j}}\right]^{a}\right\} \exp \left\{-v\left[\frac{d\left(x_{0}, \mathcal{Y}^{j}\right)}{A_{0} \delta^{j}}\right]^{a}\right\},
\end{aligned}
$$

which implies that

$$
\left\|Q_{j}(g)\right\|_{L^{\infty}(\mathcal{X})} \lesssim \frac{V\left(x_{0}, r_{0}\right)}{V_{\delta^{j}}\left(x_{0}\right)} \exp \left\{-v\left[\frac{d\left(x_{0}, \mathcal{Y}^{j}\right)}{A_{0} \delta^{j}}\right]^{a}\right\}
$$

On the other hand, from (15), we deduce that

$$
\begin{aligned}
\left\|Q_{j}(g)\right\|_{L^{1}(\mathcal{X})} & \lesssim V\left(x_{0}, r_{0}\right) \int_{\mathcal{X}} \frac{1}{V_{\delta^{j}}\left(x_{0}\right)} \exp \left\{-\frac{v}{4}\left[\frac{d\left(x, x_{0}\right)}{A_{0} \delta^{j}}\right]^{a}\right\} d \mu(x) \exp \left\{-v\left[\frac{d\left(x_{0}, \mathcal{Y}^{j}\right)}{A_{0} \delta^{j}}\right]^{a}\right\} \\
& \lesssim V\left(x_{0}, r_{0}\right) \exp \left\{-v\left[\frac{d\left(x_{0}, \mathcal{Y}^{j}\right)}{A_{0} \delta^{j}}\right]^{a}\right\} .
\end{aligned}
$$

Thus,

$\left\|Q_{j}(g)\right\|_{L^{2}(\mathcal{X})} \leq\left\|Q_{j}(g)\right\|_{L^{\infty}(\mathcal{X})}^{1 / 2}\left\|Q_{j}(g)\right\|_{L^{1}(\mathcal{X})}^{1 / 2} \lesssim V\left(x_{0}, r_{0}\right) \frac{1}{\sqrt{V_{\delta^{j}}\left(x_{0}\right)}} \exp \left\{-v\left[\frac{d\left(x_{0}, \mathcal{Y}^{j}\right)}{A_{0} \delta^{j}}\right]^{a}\right\}$, which, combined with (11), further implies that

$$
\begin{aligned}
\sum_{\left\{j \in \mathbb{Z}: \delta^{j} \geq r_{0}\right\}}\left\|Q_{j}(g)\right\|_{L^{2}(\mathcal{X})} & \lesssim V\left(x_{0}, r_{0}\right) \sum_{\left\{j \in \mathbb{Z}: \delta^{i} \geq r_{0}\right\}} \frac{1}{\sqrt{V_{\delta^{j}}\left(x_{0}\right)}} \exp \left\{-v\left[\frac{d\left(x_{0}, \mathcal{Y}^{j}\right)}{A_{0} \delta^{j}}\right]^{a}\right\} \\
& \lesssim \sqrt{V\left(x_{0}, r_{0}\right)} .
\end{aligned}
$$

(Case 2) $\delta^{j}<r_{0}$. In this case, for a fixed $x \in \mathcal{X}$, by Definition 4(v), we first write

$$
\begin{aligned}
\left|Q_{j}(g)(x)\right| & \leq \int_{\mathcal{X}}\left|Q_{j}(x, y)\right||g(y)-g(x)| d \mu(y) \\
& =\int_{B\left(x, \delta^{j}\right)}\left|Q_{j}(x, y)\right||g(y)-g(x)| d \mu(y)+\int_{\mathcal{X} \backslash B\left(x, \delta^{j}\right)} \cdots:=\mathrm{I}_{1}+\mathrm{I}_{2} .
\end{aligned}
$$

Indeed, by Definition 4(ii) and (1), we have

$$
\begin{aligned}
\mathrm{I}_{1} & \lesssim \int_{B\left(x, \delta^{j}\right)} \frac{1}{V_{\delta^{j}}(x)} \exp \left\{-\frac{v}{2}\left[\frac{d(x, y)}{\delta^{j}}\right]^{a}\right\}[d(y, x)]^{\eta}\|g\|_{\dot{C}_{b}^{\eta}(\mathcal{X})} d \mu(y) \\
& \lesssim\left(\frac{\delta^{j}}{r_{0}}\right)^{\eta} \int_{B\left(x, \delta^{j}\right)} \frac{1}{V_{\delta^{j}}(x)} \exp \left\{-\frac{v}{2}\left[\frac{d(x, y)}{\delta^{j}}\right]^{a}\right\} d \mu(y) \lesssim\left(\frac{\delta^{j}}{r_{0}}\right)^{\eta} .
\end{aligned}
$$

and

$$
\begin{aligned}
\mathrm{I}_{2} & \lesssim \sum_{k=1}^{\infty} \int_{B\left(x, 2^{k j}\right) \backslash B\left(x, 2^{k-1} \delta^{j}\right)} \frac{1}{V_{\delta^{j}}(x)} \exp \left\{-\frac{v}{2}\left[\frac{d(x, y)}{\delta^{j}}\right]^{a}\right\}[d(y, x)]^{\eta}\|g\|_{\dot{C}_{b}^{\eta}(\mathcal{X})} d \mu(y) \\
& \lesssim \sum_{k=1}^{\infty} 2^{k \eta}\left(\frac{\delta^{j}}{r_{0}}\right)^{\eta} \exp \left\{-\frac{v}{4} 2^{(k-1) a}\right\} \int_{B\left(x, 2^{k j}\right)} \frac{1}{V_{\delta^{j}}(x)} \exp \left\{-\frac{v}{4}\left[\frac{d(x, y)}{\delta^{j}}\right]^{a}\right\} d \mu(y) \\
& \lesssim \sum_{k=1}^{\infty} 2^{k \eta} \exp \left\{-\frac{v}{4} 2^{(k-1) a}\right\}\left(\frac{\delta^{j}}{r_{0}}\right)^{\eta} \lesssim\left(\frac{\delta^{j}}{r_{0}}\right)^{\eta} .
\end{aligned}
$$


Combining the estimates of $\mathrm{I}_{1}$ and $\mathrm{I}_{2}$, we obtain

$$
\left\|Q_{j}(g)\right\|_{L^{\infty}(\mathcal{X})} \lesssim\left(\frac{\delta^{j}}{r_{0}}\right)^{\eta}
$$

Now we estimate $\left\|Q_{j}(g)\right\|_{L^{1}(\mathcal{X})}$. Indeed, we know that

$$
\begin{aligned}
\left|Q_{j}(g)(x)\right| & \leq \int_{B\left(x_{0}, r_{0}\right)}\left|Q_{j}(x, y)\right||g(y)| d \mu(y) \leq\|g\|_{L^{\infty}(\mathcal{X})} \int_{B\left(x_{0}, r_{0}\right)}\left|Q_{j}(x, y)\right| d \mu(y) \\
& \leq \int_{B\left(x_{0}, r_{0}\right)}\left|Q_{j}(x, y)\right| d \mu(y) .
\end{aligned}
$$

For any fixed $x \in \mathcal{X}$, we further consider the following two cases.

Case $1 d\left(x, x_{0}\right)<2 A_{0} r_{0}$. Observe that, by Definition 4(ii) and (1),

$$
\left|Q_{j}(g)(x)\right| \lesssim 1 .
$$

Case $2 d\left(x, x_{0}\right) \geq 2 A_{0} r_{0}$. In this case, we observe that, for any $y \in B\left(x_{0}, r_{0}\right)$,

$$
d(x, y) \geq \frac{d\left(x, x_{0}\right)}{A_{0}}-d\left(y, x_{0}\right) \geq \frac{d\left(x, x_{0}\right)}{2 A_{0}}
$$

and hence, by Definition 4(ii) and (1),

$$
\begin{aligned}
\left|Q_{j}(g)(x)\right| & \lesssim \int_{B\left(x_{0}, r_{0}\right)} \frac{1}{V_{\delta^{j}}(x)} \exp \left\{-\frac{v}{2}\left[\frac{d(x, y)}{\delta^{j}}\right]^{a}\right\} d \mu(y) \\
& \lesssim \int_{B\left(x_{0}, r_{0}\right)} \frac{1}{V_{\delta^{j}}(x)} \exp \left\{-\frac{v}{2}\left[\frac{\left.d\left(x, x_{0}\right)\right)}{2 A_{0} \delta^{j}}\right]^{a}\right\} d \mu(y) \\
& \lesssim \frac{V\left(x_{0}, r_{0}\right)}{V_{\delta^{j}}\left(x_{0}\right)} \exp \left\{-\frac{v}{4}\left[\frac{\left.d\left(x, x_{0}\right)\right)}{2 A_{0} \delta^{j}}\right]^{a}\right\} .
\end{aligned}
$$

Combining Cases 1 and 2, we find that, for any $x \in \mathcal{X}$,

$$
\left|Q_{j}(g)(x)\right| \lesssim \mathbf{1}_{B\left(x_{0}, 2 A_{0} r_{0}\right)}(x)+\mathbf{1}_{\mathcal{X} \backslash B\left(x_{0}, 2 A_{0} r_{0}\right)}(x) \frac{V\left(x_{0}, r_{0}\right)}{V_{\delta^{j}}\left(x_{0}\right)} \exp \left\{-\frac{v}{4}\left[\frac{\left.d\left(x, x_{0}\right)\right)}{2 A_{0} \delta^{j}}\right]^{a}\right\},
$$

which implies that

$$
\left\|Q_{j}(g)\right\|_{L^{1}(\mathcal{X})} \lesssim V\left(x_{0}, r_{0}\right) .
$$

From this and and (17), it follows that

$$
\left\|Q_{j}(g)\right\|_{L^{2}(\mathcal{X})} \leq\left\|Q_{j}(g)\right\|_{L^{\infty}(\mathcal{X})}^{1 / 2}\left\|Q_{j}(g)\right\|_{L^{1}(\mathcal{X})}^{1 / 2} \lesssim\left(\frac{\delta^{j}}{r_{0}}\right)^{\eta / 2} \sqrt{V\left(x_{0}, r_{0}\right)},
$$

which further implies that

$$
\sum_{\left\{j \in \mathbb{Z}: \delta^{j}<r_{0}\right\}}\left\|Q_{j}(g)\right\|_{L^{2}(\mathcal{X})} \lesssim r_{0}^{-\eta / 2} \sum_{\left\{j \in \mathbb{Z}: \delta^{j}<r_{0}\right\}} \delta^{j \eta / 2} \sqrt{V\left(x_{0}, r_{0}\right)} \lesssim \sqrt{V\left(x_{0}, r_{0}\right)} .
$$

By this and (16), we conclude that

$$
\sum_{j \in \mathbb{Z}}\left\|Q_{j}(g)\right\|_{L^{2}(\mathcal{X})} \lesssim \sqrt{V\left(x_{0}, r_{0}\right)},
$$

which further completes the proof of the above claim. 
Now we begin to show that $K_{f}^{(3)}(\cdot, \cdot)$ satisfies (3) through (5). To achieve this, by (13), Definition 4(ii), (1) and (12), we find that, for any $x, y \in \mathcal{X}$ with $x \neq y$,

$$
\begin{aligned}
\left|K_{f}^{(3)}(x, y)\right| & \leq \sum_{j \in \mathbb{Z}}\left|Q_{j}(x, y)\right|\left|Q_{j}(f)(x)\right| \lesssim\|f\|_{\mathrm{BMO}(\mathcal{X})} \sum_{j \in \mathbb{Z}}\left|Q_{j}(x, y)\right| \\
& \lesssim\|f\|_{\mathrm{BMO}(\mathcal{X})} \sum_{j \in \mathbb{Z}} \frac{1}{V_{\delta^{j}}(x)} \exp \left\{-\frac{v}{2}\left[\frac{d(x, y)}{\delta^{j}}\right]^{a}\right\} \exp \left\{-\frac{v}{2}\left[\frac{d\left(x, \mathcal{Y}^{j}\right)}{\delta^{j}}\right]^{a}\right\} \\
& \lesssim\|f\|_{\mathrm{BMO}(\mathcal{X})} \frac{1}{V(x, y)} .
\end{aligned}
$$

This shows that $K_{f}^{(3)}(\cdot, \cdot)$ satisfies (3).

Then we prove that $K_{f}^{(3)}(\cdot, \cdot)$ satisfies (4). Indeed, let $x, \widetilde{x}, y \in \mathcal{X}$ with $d(x, \widetilde{x}) \leq$ $\frac{1}{2 A_{0}} d(x, y)$ and $x \neq y$. We observe that

$$
d(\widetilde{x}, y) \geq \frac{d(x, y)}{A_{0}}-d(x, \widetilde{x}) \geq \frac{d(x, y)}{2 A_{0}} .
$$

From (13), it follows that

$$
\begin{aligned}
\left|K_{f}^{(3)}(x, y)-K_{f}^{(3)}(\widetilde{x}, y)\right| & \leq \sum_{j \in \mathbb{Z}}\left|Q_{j}(f)(y)\right|\left|Q_{j}(x, y)-Q_{j}(\widetilde{x}, y)\right| \\
& \lesssim\|f\|_{\mathrm{BMO}(\mathcal{X})} \sum_{j \in \mathbb{Z}}\left|Q_{j}(x, y)-Q_{j}(\widetilde{x}, y)\right| .
\end{aligned}
$$

We further consider the following two cases.

Case (1) $d(x, \widetilde{x}) \leq \delta^{j}$. In this case, by Definition 4(iii) and (1), we have

$$
\begin{aligned}
\left|Q_{j}(x, y)-Q_{j}(\widetilde{x}, y)\right| & \lesssim\left[\frac{d(x, \widetilde{x})}{\delta^{j}}\right]^{\eta} \frac{1}{V_{\delta^{j}}(x)} \exp \left\{-\frac{v}{2}\left[\frac{d(x, y)}{\delta^{j}}\right]^{a}\right\} \exp \left\{-v\left[\frac{d\left(x, \mathcal{Y}^{j}\right)}{\delta^{j}}\right]^{a}\right\} \\
& \lesssim\left[\frac{d(x, \widetilde{x})}{d(x, y)}\right]^{\eta} \frac{1}{V_{\delta^{j}}(x)} \exp \left\{-\frac{v}{4}\left[\frac{d(x, y)}{\delta^{j}}\right]^{a}\right\} \exp \left\{-\frac{v}{4}\left[\frac{d\left(x, \mathcal{Y}^{j}\right)}{\delta^{j}}\right]^{a}\right\},
\end{aligned}
$$

which, together with (12), implies that

$$
\begin{aligned}
\sum_{\left\{j \in \mathbb{Z}: \delta^{j} \geq d(x, \widetilde{x})\right\}}\left|Q_{j}(x, y)-Q_{j}(\widetilde{x}, y)\right| \lesssim & {\left[\frac{d(x, \widetilde{x})}{d(x, y)}\right]^{\eta} \sum_{\left\{j \in \mathbb{Z}: \delta^{j} \geq d(x, \widetilde{x})\right.} \frac{1}{V_{\delta^{j}}(x)} \exp \left\{-\frac{v}{4}\left[\frac{d(x, y)}{\delta^{j}}\right]^{a}\right\} } \\
& \times \exp \left\{-\frac{v}{4}\left[\frac{d\left(x, \mathcal{Y}^{j}\right)}{\delta^{j}}\right]^{a}\right\} \\
\lesssim & {\left[\frac{d(x, \widetilde{x})}{d(x, y)}\right]^{\eta} \frac{1}{V(x, y)} . }
\end{aligned}
$$

Case (2) $d(x, \widetilde{x})>\delta^{j}$. In this case, from Definition 4(ii), (1) and (19), we deduce that

$$
\begin{aligned}
\left|Q_{j}(x, y)-Q_{j}(\widetilde{x}, y)\right| \leq & \left|Q_{j}(x, y)\right|+\left|Q_{j}(\widetilde{x}, y)\right| \\
\lesssim & \frac{1}{V_{\delta^{j}}(y)} \exp \left\{-\frac{v}{2}\left[\frac{d(x, y)}{\delta^{j}}\right]^{a}\right\} \exp \left\{-v\left[\frac{d\left(y, \mathcal{Y}^{j}\right)}{\delta^{j}}\right]^{a}\right\} \\
& +\frac{1}{V_{\delta^{j}}(y)} \exp \left\{-\frac{v}{2}\left[\frac{d(\widetilde{x}, y)}{\delta^{j}}\right]^{a}\right\} \exp \left\{-v\left[\frac{d\left(y, \mathcal{Y}^{j}\right)}{\delta^{j}}\right]^{a}\right\}
\end{aligned}
$$




$$
\begin{aligned}
& \lesssim \frac{1}{V_{\delta^{j}}(y)} \exp \left\{-\frac{v}{2}\left[\frac{d(x, y)}{2 A_{0} \delta^{j}}\right]^{a}\right\} \exp \left\{-v\left[\frac{d\left(y, \mathcal{Y}^{j}\right)}{\delta^{j}}\right]^{a}\right\} \\
& \lesssim\left[\frac{d(x, \widetilde{x})}{\delta^{j}}\right]^{\eta} \frac{1}{V_{\delta^{j}}(y)} \exp \left\{-\frac{v}{2}\left[\frac{d(x, y)}{2 A_{0} \delta^{j}}\right]^{a}\right\} \exp \left\{-v\left[\frac{d\left(y, \mathcal{Y}^{j}\right)}{\delta^{j}}\right]^{a}\right\} \\
& \lesssim\left[\frac{d(x, \widetilde{x})}{d(x, y)}\right]^{\eta} \frac{1}{V_{\delta^{j}}(y)} \exp \left\{-\frac{v}{4}\left[\frac{d(x, y)}{2 A_{0} \delta^{j}}\right]^{a}\right\} \exp \left\{-\frac{v}{4}\left[\frac{d\left(y, \mathcal{Y}^{j}\right)}{2 A_{0} \delta^{j}}\right]^{a}\right\} .
\end{aligned}
$$

Thus, by (12), we obtain

$$
\begin{aligned}
& \sum_{\left\{j \in \mathbb{Z}: \delta^{j}<d(x, \widetilde{x})\right\}}\left|Q_{j}(x, y)-Q_{j}(\widetilde{x}, y)\right| \\
& \lesssim\left[\frac{d(x, \widetilde{x})}{d(x, y)}\right]^{a} \sum_{\{j \in \mathbb{Z}: \delta j<d(x, \widetilde{x})\}} \frac{1}{V_{\delta^{j}}(y)} \exp \left\{-\frac{v}{4}\left[\frac{d(x, y)}{2 A_{0} \delta^{j}}\right]^{a}\right\} \exp \left\{-\frac{v}{4}\left[\frac{d\left(y, \mathcal{Y}^{j}\right)}{2 A_{0} \delta^{j}}\right]^{a}\right\} \\
& \lesssim\left[\frac{d(x, \widetilde{x})}{d(x, y)}\right]^{\eta} \frac{1}{V(x, y)} .
\end{aligned}
$$

Combining the Cases (1) and (2), we have

$$
\sum_{j \in \mathbb{Z}}\left|Q_{j}(x, y)-Q_{j}(\widetilde{x}, y)\right| \lesssim\left[\frac{d(x, \widetilde{x})}{d(x, y)}\right]^{\eta} \frac{1}{V(x, y)},
$$

which further proves that $K_{f}^{(3)}(\cdot, \cdot)$ satisfies (4). By the arguments similar to those used in the proof of (20), we conclude that

$$
\sum_{\{j \in \mathbb{Z}\}}\left|Q_{j}(y, x)-Q_{j}(y, \widetilde{x})\right| \lesssim\left[\frac{d(x, \widetilde{x})}{d(x, y)}\right]^{\eta} \frac{1}{V(x, y)} .
$$

We further show that $K_{f}^{(3)}(\cdot, \cdot)$ satisfies (5). Indeed, let $x, \widetilde{x}, y \in \mathcal{X}$ with $d(x, \widetilde{x}) \leq$ $\frac{1}{2 A_{0}} d(x, y)$ and $x \neq y$. From (13), Definition 4(v), (1) and (21), we deduce that

$$
\begin{aligned}
& \left|K_{f}^{(3)}(y, x)-K_{f}^{(3)}(y, \widetilde{x})\right| \\
& \leq \sum_{j \in \mathbb{Z}}\left|Q_{j}(y, x) Q_{j}(f)(x)-Q_{j}(y, \widetilde{x}) Q_{j}(f)(\widetilde{x})\right| \\
& \leq \sum_{j \in \mathbb{Z}}\left|Q_{j}(y, x)-Q_{j}(y, \widetilde{x})\right|\left|Q_{j}(f)(x)\right|+\sum_{j \in \mathbb{Z}}\left|Q_{j}(y, \widetilde{x})\right|\left|Q_{j}(f)(x)-Q_{j}(f)(\widetilde{x})\right| \\
& \quad \lesssim\|f\|_{\mathrm{BMO}(\mathcal{X})} \sum_{j \in \mathbb{Z}}\left|Q_{j}(y, x)-Q_{j}(y, \widetilde{x})\right| \\
& \quad+\sum_{j \in \mathbb{Z}}\left|Q_{j}(y, \widetilde{x})\right| \int_{\mathcal{X}}\left|Q_{j}(x, z)-Q_{j}(\widetilde{x}, z)\right|\left|f(z)-m_{B(x, \delta j)}(f)\right| d \mu(z) \\
& \quad \lesssim\|f\|_{\mathrm{BMO}(\mathcal{X})}\left[\frac{d(x, \widetilde{x})}{d(y, x)}\right]^{\eta} \frac{1}{V(x, y)}+\mathrm{A},
\end{aligned}
$$

where

$$
\mathrm{A}:=\sum_{j \in \mathbb{Z}}\left|Q_{j}(y, \widetilde{x})\right| \int_{\mathcal{X}}\left|Q_{j}(x, z)-Q_{j}(\widetilde{x}, z)\right|\left|f(z)-m_{B(x, \delta j)}(f)\right| d \mu(z) .
$$

To estimate A, we deal with the following two cases. 
Case (i) $d(x, \widetilde{x}) \leq \delta^{j}$. By (ii) and (iii) of Definition 4, (1), (19), some arguments similar to those used in the proof of ([62], (2.3)) and (12), we conclude that

$$
\begin{aligned}
\mathrm{A} \lesssim & \sum_{j \in \mathbb{Z}} \frac{1}{V_{\delta^{j}}(y)} \exp \left\{-\frac{v}{2}\left[\frac{d(\widetilde{x}, y)}{\delta^{j}}\right]^{a}\right\} \exp \left\{-v\left[\frac{d\left(y, \mathcal{Y}^{j}\right)}{\delta^{j}}\right]^{a}\right\} \\
& \times \int_{\mathcal{X}}\left[\frac{d(x, \widetilde{x})}{\delta^{j}}\right]^{\eta} \frac{1}{\sqrt{V_{\delta^{j}}(x) V_{\delta^{j}}(\widetilde{x})}} \exp \left\{-\frac{v}{4}\left[\frac{d(x, z)}{\delta^{j}}\right]^{a}\right\} \exp \left\{-\frac{v}{4}\left[\frac{d(\widetilde{x}, z)}{\delta^{j}}\right]^{a}\right\} \\
& \times\left|f(z)-m_{B\left(x, \delta^{j}\right)}(f)\right| d \mu(z) \\
\lesssim & \sum_{j \in \mathbb{Z}} \frac{1}{V_{\delta^{j}}(y)} \exp \left\{-\frac{v}{2}\left[\frac{d(x, y)}{2 A_{0} \delta^{j}}\right]^{a}\right\} \exp \left\{-v\left[\frac{d\left(y, \mathcal{Y}^{j}\right)}{\delta^{j}}\right]^{a}\right\} \\
& \times \int_{\mathcal{X}}\left[\frac{d(x, \widetilde{x})}{\delta^{j}}\right]^{\eta} \frac{1}{\sqrt{V_{\delta^{j}}(x) V_{\delta^{j}}(\widetilde{x})}} \exp \left\{-\frac{v}{8}\left[\frac{d(x, z)}{\delta^{j}}\right]^{a}\right\} \exp \left\{-\frac{v}{8}\left[\frac{d(x, \widetilde{x})}{A_{0} \delta^{j}}\right]^{a}\right\} \\
& \times\left.\right|_{f(z)-m_{B\left(x, \delta^{j}\right)}(f) \mid d \mu(z)} \sum_{j \in \mathbb{Z}} \frac{1}{V_{\delta^{j}}(y)} \exp \left\{-\frac{v}{4}\left[\frac{d(x, y)}{2 A_{0} \delta^{j}}\right]^{a}\right\} \exp \left\{-v\left[\frac{d\left(y, \mathcal{Y}^{j}\right)}{\delta^{j}}\right]^{a}\right\} \\
& \times \int_{\mathcal{X}}\left[\frac{d(x, \widetilde{x})}{d(x, y)}\right]^{\eta} \frac{1}{V_{\delta^{j}}(x)} \exp \left\{-\frac{v}{8}\left[\frac{d(x, z)}{\delta^{j}}\right]^{a}\right\}\left|f(z)-m_{B(x, \delta j)}(f)\right| d \mu(z) \\
\lesssim & \|f\|_{\mathrm{BMO}(\mathcal{X})}\left[\frac{d(x, \widetilde{x})}{d(x, y)}\right]^{\eta} \sum_{j \in \mathbb{Z}} \frac{1}{V_{\delta^{j}}(y)} \exp \left\{-\frac{v}{4}\left[\frac{d(x, y)}{2 A_{0} \delta^{j}}\right]^{a}\right\} \exp \left\{-\frac{v}{4}\left[\frac{d\left(y, \mathcal{Y}^{j}\right)}{2 A_{0} \delta^{j}}\right]^{a}\right\} \\
\lesssim & \|f\|_{\mathrm{BMO}(\mathcal{X})}\left[\frac{d(x, \widetilde{x})}{d(y, x)}\right]^{\eta} \frac{1}{V(x, y)} .
\end{aligned}
$$

Case (ii) $d(x, \widetilde{x})>\delta^{j}$. From Definition 4(ii), (1), (19), some arguments similar to those used in the proof of ([62], (2.3)) and (12), we deduce that

$$
\begin{aligned}
\mathrm{A} \lesssim & \sum_{j \in \mathbb{Z}} \frac{1}{V_{\delta^{j}}(y)} \exp \left\{-\frac{v}{2}\left[\frac{d(\widetilde{x}, y)}{\delta^{j}}\right]^{a}\right\} \exp \left\{-v\left[\frac{d\left(y, \mathcal{Y}^{j}\right)}{\delta^{j}}\right]^{a}\right\} \\
& \times \int_{\mathcal{X}}\left[\frac{d(x, \widetilde{x})}{\delta^{j}}\right]^{\eta}\left[\frac{1}{V_{\delta^{j}}(x)} \exp \left\{-\frac{v}{2}\left[\frac{d(x, z)}{\delta^{j}}\right]^{a}\right\}+\frac{1}{V_{\delta^{j}}(z)} \exp \left\{-\frac{v}{2}\left[\frac{d(\widetilde{x}, z)}{\delta^{j}}\right]^{a}\right\}\right] \\
& \times\left|f(z)-m_{B\left(x, \delta^{j}\right)}(f)\right| d \mu(z) \\
\lesssim & \sum_{j \in \mathbb{Z}} \frac{1}{V_{\delta^{j}}(y)} \exp \left\{-\frac{v}{4}\left[\frac{d(\widetilde{x}, y)}{\delta^{j}}\right]^{a}\right\} \exp \left\{-\frac{v}{4}\left[\frac{d(x, y)}{2 A_{0} \delta^{j}}\right]^{a}\right\} \exp \left\{-v\left[\frac{d\left(y, \mathcal{Y}^{j}\right)}{\delta^{j}}\right]^{a}\right\} \\
& \times \int_{\mathcal{X}}\left[\frac{d(x, \widetilde{x})}{\delta^{j}}\right]^{\eta}\left[\frac{1}{V_{\delta^{j}}(x)} \exp \left\{-\frac{v}{2}\left[\frac{d(x, z)}{\delta^{j}}\right]^{a}\right\}+\frac{1}{V_{\delta^{j}}(z)} \exp \left\{-\frac{v}{2}\left[\frac{d(\widetilde{x}, z)}{\delta^{j}}\right]^{a}\right\}\right] \\
& \times\left|f(z)-m_{B\left(x, \delta^{j}\right)}(f)\right| d \mu(z) \\
\vdots & \left.\frac{d(x, \widetilde{x})}{d(y, x)}\right]^{\eta} \sum_{j \in \mathbb{Z}} \frac{1}{V_{\delta^{j}}(y)} \exp \left\{-\frac{v}{8}\left[\frac{d(x, y)}{2 A_{0} \delta^{j}}\right]^{a}\right\} \exp \left\{-v\left[\frac{d\left(y, \mathcal{Y}^{j}\right)}{\delta^{j}}\right]^{a}\right\} \\
& \times \int_{\mathcal{X}} \frac{1}{V_{\delta^{j}}(x)} \exp \left\{-\frac{v}{2}\left[\frac{d(x, z)}{\delta^{j}}\right]^{a}\right\}\left|f(z)-m_{B(x, \delta j)}(f)\right| d \mu(z) \\
& +\left[\frac{d(x, \widetilde{x})}{d(y, x)}\right]^{\eta} \sum_{j \in \mathbb{Z}} \frac{1}{V_{\delta^{j}}(y)} \exp \left\{-\frac{v}{16}\left[\frac{d(x, y)}{2 A_{0} \delta^{j}}\right]^{a}\right\} \exp \left\{-v\left[\frac{d\left(y, \mathcal{Y}^{j}\right)}{\delta^{j}}\right]^{a}\right\}
\end{aligned}
$$




$$
\begin{aligned}
& \times \int_{\mathcal{X}} \frac{1}{V_{\delta^{j}}(z)} \exp \left\{-\frac{v}{16}\left[\frac{d(x, z)}{2 A_{0}^{2} \delta^{j}}\right]^{a}\right\}\left|f(z)-m_{B\left(x, \delta^{j}\right)}(f)\right| d \mu(z) \\
\lesssim & \|f\|_{\mathrm{BMO}(\mathcal{X})}\left[\frac{d(x, \widetilde{x})}{d(x, y)}\right]^{\eta} \sum_{j \in \mathbb{Z}} \frac{1}{V_{\delta^{j}}(y)} \exp \left\{-\frac{v}{8}\left[\frac{d(x, y)}{2 A_{0} \delta^{j}}\right]^{a}\right\} \exp \left\{-\frac{v}{8}\left[\frac{d\left(y, \mathcal{Y}^{j}\right)}{2 A_{0} \delta^{j}}\right]^{a}\right\} \\
\lesssim & \|f\|_{\mathrm{BMO}(\mathcal{X})}\left[\frac{d(x, \widetilde{x})}{d(y, x)}\right]^{\eta} \frac{1}{V(x, y)} .
\end{aligned}
$$

Combining Cases (i) and (ii), we know that $K_{f}^{(3)}(\cdot, \cdot)$ satisfies (5). These complete the proof of (3) through (5) for $K_{f}^{(3)}(\cdot, \cdot)$.

Next we show that $K_{f}^{(3)}(1),\left(K_{f}^{(3)}\right)^{*}(1) \in \operatorname{BMO}(\mathcal{X})$. Obviously, $K_{f}^{(3)}(1)=0 \in$ $\mathrm{BMO}(\mathcal{X})$. Now we prove that $\left(K_{f}^{(3)}\right)^{*}(1) \in \operatorname{BMO}(\mathcal{X})$. It is easy to see that

$$
\left(K_{f}^{(3)}\right)^{*}(x, y)=K_{f}^{(3)}(y, x)=\sum_{j \in \mathbb{Z}} Q_{j}(y, x) Q_{j}(f)(y) .
$$

For any $h \in \dot{C}_{b}^{\eta}(\mathcal{X})$ with supp $h \subset B\left(x_{0}, r_{0}\right)$ and any $N \in \mathbb{N}$, choose $\eta_{N} \in C_{b}^{\eta}(\mathcal{X})$ with $\eta_{N} \equiv 1$ on $B\left(x_{0}, 2 A_{0} N r_{0}\right)$, supp $\left(\eta_{N}\right) \subset B\left(x_{0}, 4 A_{0} N r_{0}\right)$, and $0 \leq \eta_{N} \leq 1$. We write

$$
\left\langle\left(K_{f}^{(3)}\right)^{*}(1), h\right\rangle=\left\langle\left(K_{f}^{(3)}\right)^{*}\left(\eta_{N}\right), h\right\rangle+\int_{\mathcal{X}}\left[1-\eta_{N}(x)\right] K_{f}^{(3)}(h)(x) d \mu(x)=: \mathrm{I}_{N}+\mathrm{II}_{N} .
$$

By $\int_{\mathcal{X}} h(y) d \mu(y)=0$, we conclude that

$$
\begin{aligned}
\mathrm{II}_{N} & =\int_{\mathcal{X}}\left[1-\eta_{N}(x)\right] \int_{\mathcal{X}} K_{f}^{(3)}(x, y) h(y) d \mu(y) d \mu(x) \\
& =\int_{\mathcal{X}} \int_{B\left(x_{0}, r_{0}\right)} K_{f}^{(3)}(x, y)\left[1-\eta_{N}(x)\right] h(y) d \mu(y) d \mu(x) \\
& =\int_{\mathcal{X}} \int_{B\left(x_{0}, r_{0}\right)}\left[K_{f}^{(3)}(x, y)-K_{f}^{(3)}\left(x, x_{0}\right)\right]\left[1-\eta_{N}(x)\right] h(y) d \mu(y) d \mu(x) .
\end{aligned}
$$

We observe that if $d\left(y, x_{0}\right)<r_{0}$ and $d\left(x, x_{0}\right) \geq 2 A_{0} N r_{0}$, then $d\left(x, x_{0}\right) \geq 2 A_{0} N r_{0} \geq$ $2 A_{0} d\left(y, x_{0}\right)$, which implies that

$$
\begin{aligned}
\left|\mathrm{II}_{N}\right| & \leq \int_{\mathcal{X} \backslash B\left(x_{0}, 2 A_{0} N r_{0}\right)} \int_{B\left(x_{0}, r_{0}\right)}\left|K_{f}^{(3)}(x, y)-K_{f}^{(3)}\left(x, x_{0}\right)\right|\left|1-\eta_{N}(x)\right||h(y)| d \mu(y) d \mu(x) \\
& \lesssim\|h\|_{L^{\infty}(\mathcal{X})} \int_{\mathcal{X} \backslash B\left(x_{0}, 2 A_{0} N r_{0}\right)} \int_{B\left(x_{0}, r_{0}\right)}\left[\frac{d\left(y, x_{0}\right)}{d\left(x, x_{0}\right)}\right]^{\eta} \frac{1}{V\left(x, x_{0}\right)} d \mu(y) d \mu(x) \\
& \lesssim\|h\|_{L^{\infty}(\mathcal{X})} r_{0}^{\eta} V\left(x_{0}, r_{0}\right) \int_{\mathcal{X} \backslash B\left(x_{0}, 2 A_{0} N r_{0}\right)}\left[\frac{1}{d\left(x, x_{0}\right)}\right]^{\eta} \frac{1}{V\left(x, x_{0}\right)} d \mu(x) \rightarrow 0, \quad \text { as } \quad N \rightarrow \infty .
\end{aligned}
$$

Then we need to prove $\lim _{N \rightarrow \infty} \mathrm{I}_{N}=\langle f, h\rangle$. Indeed, for any $h \in \dot{C}_{b}^{\eta}(\mathcal{X})$, observe that, by ([29], Corollary 3.14) and the boundedness of $Q_{j}$ on $L^{2}(\mathcal{X})$, we know that $Q_{j}(f) \eta_{N} \in$ $L^{2}(\mathcal{X})$, which, combined with the assumptions (a) and (b) in Theorem 3 , the fact that $h$ is a multiple of a $(1,2)$-atom, and the Lebesgue dominated convergence theorem, further implies that

$$
\begin{aligned}
\lim _{N \rightarrow \infty}\left\langle\left(K_{f}^{(3)}\right)^{*}\left(\eta_{N}\right), h\right\rangle & =\lim _{N \rightarrow \infty} \sum_{j \in \mathbb{Z}}\left\langle Q_{j}^{*}\left(Q_{j}(f) \eta_{N}\right), h\right\rangle=\lim _{N \rightarrow \infty} \sum_{j \in \mathbb{Z}}\left\langle Q_{j}(f) \eta_{N}, Q_{j}(h)\right\rangle \\
& =\lim _{N \rightarrow \infty} \sum_{j \in \mathbb{Z}}\left\langle\eta_{N}, Q_{j}(f) Q_{j}(h)\right\rangle=\lim _{N \rightarrow \infty}\left\langle\eta_{N}, \sum_{j \in \mathbb{Z}} Q_{j}(f) Q_{j}(h)\right\rangle
\end{aligned}
$$




$$
\begin{aligned}
& =\left\langle 1, \sum_{j \in \mathbb{Z}} Q_{j}(f) Q_{j}(h)\right\rangle=\sum_{j \in \mathbb{Z}}\left\langle Q_{j}(f), Q_{j}(h)\right\rangle \\
& =\sum_{j \in \mathbb{Z}}\left\langle f, Q_{j}^{*} Q_{j}(h)\right\rangle=\left\langle f, \sum_{j \in \mathbb{Z}} Q_{j}(h)\right\rangle=\langle f, h\rangle,
\end{aligned}
$$

where in the fifth inequality of this equation, we need to show that the series $\sum_{j \in \mathbb{Z}} Q_{j}(f) Q_{j}(h)$ absolutely converges in $L^{1}(\mathcal{X})$. Indeed, from (13), ([62], (2.4)) and the fact that $C_{b}^{\eta}(\mathcal{X}) \subset$ $\mathcal{G}\left(x_{1}, r, \eta, \vartheta\right)$ for any given $x_{1} \in \mathcal{X}$ and $r, \vartheta \in(0, \infty)$ (see ([33], p. 19)), it follows that

$$
\begin{aligned}
\left\|\sum_{j \in \mathbb{Z}}\left|Q_{j}(f) Q_{j}(h)\right|\right\|_{L^{1}(\mathcal{X})} & \leq \sum_{j \in \mathbb{Z}}\left\|Q_{j}(f) Q_{j}(h)\right\|_{L^{1}(\mathcal{X})} \leq \sum_{j \in \mathbb{Z}}\left\|Q_{j}(f)\right\|_{L^{\infty}(\mathcal{X})}\left\|Q_{j}(h)\right\|_{L^{1}(\mathcal{X})} \\
& \lesssim\|f\|_{\mathrm{BMO}(\mathcal{X})} \sum_{j \in \mathbb{Z}}\left\|Q_{j}(h)\right\|_{L^{1}(\mathcal{X})} \lesssim\|f\|_{\mathrm{BMO}(\mathcal{X})}\|h\|_{\mathcal{G}\left(x_{1}, r, \eta, \vartheta\right)}<\infty,
\end{aligned}
$$

which proves the desired result. This shows $\lim _{N \rightarrow \infty} \mathrm{I}_{N}=\langle f, h\rangle$, which, together with the estimate of $\mathrm{II}_{N}$, implies that $\left(K_{f}^{(3)}\right)^{*}(1)=f$ on $\left(C_{b}^{\eta}(\mathcal{X})\right)^{\prime}$ and hence $\left(K_{f}^{(3)}\right)^{*}(1)=f \in$ $\operatorname{BMO}(\mathcal{X})$.

Moreover, from the $T(1)$ theorem (see Lemma 3) ([32], Theorem 12.2), we deduce that $K_{f}^{(3)}$ is bounded on $L^{2}(\mathcal{X})$. Then, by the boundedness of the Calderón-Zygmund operator (see, for example, ([27], Theorem 2.4 in Chapter III), ([28], p. 599), ([35], Theorem 1.12), and ([58], Theorem 3.4)), we find that (i)-(iv) of Theorem 3 hold true.

Now we begin to show (v) and (vi) of Theorem 3 . To this end, we temporarily fix a $g \in L^{\infty}(\mathcal{X})$. From the fact that $g \in L^{\infty}(\mathcal{X}) \subset \operatorname{BMO}(\mathcal{X})$, and the arguments used in the proof of (i)-(iv) of Theorem 3, it follows that the kernel of the operator $K_{g}^{(3)}(\cdot):=\Pi(\cdot, g)$, defined by setting, for any $(x, y) \in \mathcal{X} \times \mathcal{X}$,

$$
K_{g}^{(3)}(x, y):=\sum_{j \in \mathbb{Z}} Q_{j}(x, y) Q_{j}(g)(y)
$$

satisfies (3) through (5) and $\operatorname{WBP}(\eta)$ with $\|f\|_{\mathrm{BMO}(\mathcal{X})}$ replaced by $\|g\|_{L^{\infty}(\mathcal{X})}, K_{g}^{(3)}(1)=0 \in$ $\operatorname{BMO}(\mathcal{X}),\left(K_{g}^{(3)}\right)^{*}(1)=g \in L^{\infty}(\mathcal{X}) \subset \operatorname{BMO}(\mathcal{X})$ and $K_{g}^{(3)}$ is bounded on $L^{2}(\mathcal{X})$.

Thus, $K_{g}^{(3)}$ is an $\eta$-Calderón-Zygmund operator, which, combined with the fact that $\left(K_{g}^{(3)}\right)^{*}(1)=g \in L^{\infty}(\mathcal{X}) \subset \operatorname{BMO}(\mathcal{X})$ and the $T(1)$ theorem (see Lemma 3) and ([27], Theorem 2.4 in Chapter III), further completes the proof of (v) and (vi) of Theorem 3 and hence of Theorem 3.

Proof of Theorem 4. Similar to the proof of Theorem 3, without loss of generality, we may assume that the $\operatorname{sum} \sum_{j \in \mathbb{Z}}$ in $\Pi_{1}(f, g)$ is a finite $\operatorname{sum} \sum_{j=-N}^{N}$ for any fixed $N \in \mathbb{N}$.

We first prove (i) through (iv) of Theorem 4. Fix $f \in L^{\infty}(\mathcal{X})$, we consider the operator $K_{f}^{(1)}$ and its kernel, which is still denoted by $K_{f}^{(1)}$, defined by setting, for any $x \in \mathcal{X}$,

$$
K_{f}^{(1)}(g)(x):=\Pi_{1}(f, g)(x)=\sum_{j \in \mathbb{Z}} P_{j}(f)(x) Q_{j}(g)(x)=\int_{\mathcal{X}} K_{f}^{(1)}(x, y) g(y) d \mu(y),
$$

where $K_{f}^{(1)}(x, y)=\sum_{j \in \mathbb{Z}} Q_{j}(x, y) P_{j}(f)(x)$. 
Now we show that $K_{f}^{(1)}(\cdot, \cdot)$ satisfies (3) through (5). To this end, we first prove that $K_{f}^{(1)}(\cdot, \cdot)$ satisfies (3). From Remark 2(ii), it follows that there exists a positive constant $C$ such that, for any $f \in L^{\infty}(\mathcal{X})$,

$$
\sup _{j \in \mathbb{Z}}\left\|P_{j}(f)\right\|_{L^{\infty}(\mathcal{X})} \leq C\|f\|_{L^{\infty}(\mathcal{X})},
$$

which, together with (13) and some arguments used in the proof of (18), further implies that, for any $x, y \in \mathcal{X}$,

$$
\left|K_{f}^{(1)}(x, y)\right| \leq \sum_{j \in \mathbb{Z}}\left|Q_{j}(x, y)\left\|P_{j}(f)(x)\left|\lesssim\|f\|_{L^{\infty}(\mathcal{X})} \sum_{j \in \mathbb{Z}}\right| Q_{j}(x, y) \mid \lesssim\right\| f \|_{L^{\infty}(\mathcal{X})} \frac{1}{V(x, y)},\right.
$$

which completes the proof of (3) for $K_{f}^{(1)}(\cdot, \cdot)$.

Then we prove that $K_{f}^{(1)}(\cdot, \cdot)$ satisfies (4). Let $x, \widetilde{x}, y \in \mathcal{X}, d(x, \widetilde{x}) \leq \frac{1}{2 A_{0}} d(x, y)$ with $x \neq y$. We write

$$
\begin{aligned}
\left|K_{f}^{(1)}(x, y)-K_{f}^{(1)}(\widetilde{x}, y)\right| \leq & \sum_{j \in \mathbb{Z}}\left|Q_{j}(x, y)-Q_{j}(\widetilde{x}, y)\right|\left|P_{j}(f)(x)\right| \\
& +\sum_{j \in \mathbb{Z}}\left|Q_{j}(\widetilde{x}, y)\right|\left|P_{j}(f)(x)-P_{j}(f)(\widetilde{x})\right| \\
= & : \mathrm{A}_{1}+\mathrm{A}_{2} .
\end{aligned}
$$

From (20) and (23), we deduce that

$$
\mathrm{A}_{1} \lesssim\|f\|_{L^{\infty}(\mathcal{X})} \sum_{j \in \mathbb{Z}}\left|Q_{j}(x, y)-Q_{j}(\widetilde{x}, y)\right| \lesssim\|f\|_{L^{\infty}(\mathcal{X})}\left[\frac{d(x, \widetilde{x})}{d(x, y)}\right]^{\eta} \frac{1}{V(x, y)}
$$

Moreover, by the fact that $L^{\infty}(\mathcal{X}) \subset \mathrm{BMO}(\mathcal{X})$ and some arguments similar to those used in the proof of (22), we know that

$$
\begin{aligned}
\mathrm{A}_{2} & \lesssim \sum_{j \in \mathbb{Z}}\left|Q_{j}(\widetilde{x}, y)\right| \int_{\mathcal{X}}\left|P_{j}(x, z)-P_{j}(\widetilde{x}, z)\right|\left|f(z)-m_{B(x, \delta j)}(f)\right| d \mu(z) \\
& \lesssim\|f\|_{\mathrm{BMO}(\mathcal{X})}\left[\frac{d(x, \widetilde{x})}{d(y, x)}\right]^{\eta} \frac{1}{V(x, y)} \lesssim\|f\|_{L^{\infty}(\mathcal{X})}\left[\frac{d(x, \widetilde{x})}{d(y, x)}\right]^{\eta} \frac{1}{V(x, y)},
\end{aligned}
$$

which completes the proof of (4) for $K_{f}^{(1)}(\cdot, \cdot)$.

Now we prove that $K_{f}^{(1)}(\cdot, \cdot)$ satisfies (5). Let $x, \widetilde{x}, y \in \mathcal{X}, d(x, \widetilde{x}) \leq \frac{1}{2 A_{0}} d(x, y)$ with $x \neq y$. From (18) and (21), it follows that

$$
\begin{aligned}
\left|K_{f}^{(1)}(y, x)-K_{f}^{(1)}(y, \widetilde{x})\right| & \leq \sum_{j \in \mathbb{Z}}\left|Q_{j}(y, x)-Q_{j}(y, \widetilde{x})\right|\left|P_{j}(f)(y)\right| \\
& \lesssim\|f\|_{L^{\infty}(\mathcal{X})} \sum_{j \in \mathbb{Z}}\left|Q_{j}(y, x)-Q_{j}(y, \widetilde{x})\right| \\
& \lesssim\|f\|_{L^{\infty}(\mathcal{X})}\left[\frac{d(x, \widetilde{x})}{d(y, x)}\right]^{\eta} \frac{1}{V(x, y)},
\end{aligned}
$$

which completes the proof of (5) for $K_{f}^{(1)}(\cdot, \cdot)$. This completes the proof of (3) through (5) for $K_{f}^{(1)}(\cdot, \cdot)$. 
Then we claim that $K_{f}^{(1)}$ has $\operatorname{WBP}(\eta)$ and hence maps from $C_{b}^{\eta}(\mathcal{X})$ into $\left(C_{b}^{\eta}(\mathcal{X})\right)^{\prime}$. Indeed, let $g, h \in C_{b}^{\eta}(\mathcal{X})$, supported on some ball $B\left(x_{0}, r_{0}\right)$ with $x_{0} \in \mathcal{X}$ and $r_{0} \in(0, \infty)$, normalized by

$$
\|g\|_{L^{\infty}(\mathcal{X})}+r_{0}^{\eta}\|g\|_{\dot{C}_{b}^{\eta}(\mathcal{X})} \leq 1 \quad \text { and } \quad\|h\|_{L^{\infty}(\mathcal{X})}+r_{0}^{\eta}\|h\|_{\dot{C}_{b}^{\eta}(\mathcal{X})} \leq 1 .
$$

Then, by (23), the Hölder inequality and (14), we conclude that

$$
\begin{aligned}
\left|\left\langle K_{f}^{(1)}(g), h\right\rangle\right| & =\left|\left\langle\Pi_{1}(f, g), h\right\rangle\right|=\left|\int_{\mathcal{X}} \Pi_{1}(f, g)(x) h(x) d \mu(x)\right| \\
& \leq \sum_{j \in \mathbb{Z}} \int_{\mathcal{X}}\left|P_{j}(f)(x) \| Q_{j}(g)(x)\right||h(x)| d \mu(x) \\
& \lesssim\|f\|_{L^{\infty}(\mathcal{X})} \sum_{j \in \mathbb{Z}} \int_{\mathcal{X}}\left|Q_{j}(g)(x)\right||h(x)| d \mu(x) \\
& \lesssim\|f\|_{L^{\infty}(\mathcal{X})} \sum_{j \in \mathbb{Z}}\left\|Q_{j}(g)\right\|_{L^{2}(\mathcal{X})}\|h\|_{L^{2}(\mathcal{X})} \\
& \lesssim\|f\|_{L^{\infty}(\mathcal{X})}\left[V\left(x_{0}, r_{0}\right)\right]^{1 / 2} \sum_{j \in \mathbb{Z}}\left\|Q_{j}(g)\right\|_{L^{2}(\mathcal{X})} \lesssim\|f\|_{L^{\infty}(\mathcal{X})} V\left(x_{0}, r_{0}\right) .
\end{aligned}
$$

Next we show that $K_{f}^{(1)}(1),\left(K_{f}^{(1)}\right)^{*}(1) \in \operatorname{BMO}(\mathcal{X})$. Obviously, $K_{f}^{(1)}(1)=0 \in$ $\mathrm{BMO}(\mathcal{X})$. Now we prove that $\left(K_{f}^{(1)}\right)^{*}(1)=0 \in \mathrm{BMO}(\mathcal{X})$. It is easy to see that

$$
\left(K_{f}^{(1)}\right)^{*}(x, y)=K_{f}^{(1)}(y, x)=\sum_{j \in \mathbb{Z}} Q_{j}(y, x) P_{j}(f)(y) .
$$

For any $h \in \dot{C}_{b}^{\eta}(\mathcal{X})$ with supp $h \subset B\left(x_{0}, r_{0}\right)$ and any $N \in \mathbb{N}$, choose $\eta_{N} \in C_{b}^{\eta}(\mathcal{X})$ with $\eta_{N} \equiv 1$ on $B\left(x_{0}, 2 A_{0} N r_{0}\right)$, supp $\left(\eta_{N}\right) \subset B\left(x_{0}, 4 A_{0} N r_{0}\right)$ and $0 \leq \eta_{N} \leq 1$. We write

$$
\left\langle\left(K_{f}^{(1)}\right)^{*}(1), h\right\rangle=\left\langle\left(K_{f}^{(1)}\right)^{*}\left(\eta_{N}\right), h\right\rangle+\int_{\mathcal{X}}\left[1-\eta_{N}(x)\right] K_{f}^{(1)}(h) d \mu(x)=: \mathrm{I}_{N}+\mathrm{II}_{N} .
$$

By the same arguments used in the proof of $\left(K_{f}^{(3)}\right)^{*}(1) \in \operatorname{BMO}(\mathcal{X})$, we conclude that $\lim _{N \rightarrow \infty} \mathrm{II}_{N}=0$. Then we show that $\lim _{N \rightarrow \infty} \mathrm{I}_{N}=0$. Indeed, for any $h \in \dot{C}_{b}^{\eta}(\mathcal{X})$, observe that, by ([29], Corollary 3.14) and the boundedness of $P_{j}$ on $L^{2}(\mathcal{X})$, we know that $P_{j}(f) \eta_{N} \in L^{2}(\mathcal{X})$, which, combined with the assumption (10), and the Lebesgue dominated convergence theorem, further implies that

$$
\begin{aligned}
\lim _{N \rightarrow \infty}\left\langle\left(K_{f}^{(1)}\right)^{*}\left(\eta_{N}\right), h\right\rangle & =\lim _{N \rightarrow \infty} \sum_{j \in \mathbb{Z}}\left\langle Q_{j}^{*}\left(P_{j}(f) \eta_{N}\right), h\right\rangle=\lim _{N \rightarrow \infty} \sum_{j \in \mathbb{Z}}\left\langle P_{j}(f) \eta_{N}, Q_{j}(h)\right\rangle \\
& =\lim _{N \rightarrow \infty} \sum_{j \in \mathbb{Z}}\left\langle\eta_{N}, P_{j}(f) Q_{j}(h)\right\rangle=\lim _{N \rightarrow \infty}\left\langle\eta_{N}, \sum_{j \in \mathbb{Z}} P_{j}(f) Q_{j}(h)\right\rangle \\
& =\left\langle 1, \sum_{j \in \mathbb{Z}} P_{j}(f) Q_{j}(h)\right\rangle=\left\langle 1, \Pi_{1}(f, h)\right\rangle=0,
\end{aligned}
$$

where in the third to the last inequality of this equation, we have used the fact that the series $\sum_{j \in \mathbb{Z}} P_{j}(f) Q_{j}(h)$ absolutely converges in $L^{1}(\mathcal{X})$, which is similar to that of $\sum_{j \in \mathbb{Z}} Q_{j}(f) Q_{j}(h)$.

This shows $\lim _{N \rightarrow \infty} \mathrm{I}_{N}=0$, which, together with the estimate of $\mathrm{II}_{N}$, implies that $\left(K_{f}^{(1)}\right)^{*}(1)=0$ on $\left(C_{b}^{\eta}(\mathcal{X})\right)^{\prime}$ and hence $\left(K_{f}^{(1)}\right)^{*}(1)=0 \in \operatorname{BMO}(\mathcal{X})$. 
Moreover, from the $T(1)$ theorem (see Lemma 3), we deduce that $K_{f}^{(1)}$ is bounded on $L^{2}(\mathcal{X})$. Then, by the boundedness of the Calderón-Zygmund operator (see, for instance, ([27], Theorem 2.4 in Chapter III)), we find that (i) through (iv) of Theorem 4 hold true.

Now we begin to prove (v) and (vi) of Theorem 4. To this end, we temporarily fix a $g \in L^{\infty}(\mathcal{X})$. By the fact that $L^{\infty}(\mathcal{X}) \subset \operatorname{BMO}(\mathcal{X})$ and checking the proofs of Theorem 3 and (i) and (ii) of Theorem 4 carefully, we conclude that there exists a positive constant $C$ such that, for any $f \in L^{\infty}(\mathcal{X}), g \in L^{2}(\mathcal{X})$, and $N \in \mathbb{N}$,

$$
\left\|\Pi_{1}^{(N)}(f, g)\right\|_{L^{2}(\mathcal{X})}+\left\|\Pi_{3}^{(N)}(f, g)\right\|_{L^{2}(\mathcal{X})} \leq C\|f\|_{L^{\infty}(\mathcal{X})}\|g\|_{L^{2}(\mathcal{X})},
$$

which, further implies that, for any $g \in L^{\infty}(\mathcal{X})$ and $f \in L^{2}(\mathcal{X})$,

$$
\begin{aligned}
\left\|\Pi_{2}^{(N)}(f, g)\right\|_{L^{2}(\mathcal{X})}+\left\|\Pi_{3}^{(N)}(f, g)\right\|_{L^{2}(\mathcal{X})} & =\left\|\Pi_{1}^{(N)}(g, f)\right\|_{L^{2}(\mathcal{X})}+\left\|\Pi_{3}^{(N)}(g, f)\right\|_{L^{2}(\mathcal{X})} \\
& \lesssim\|g\|_{L^{\infty}(\mathcal{X})}\|f\|_{L^{2}(\mathcal{X})} .
\end{aligned}
$$

From this and Theorem 1 , we deduce that $\Pi_{1}(f, g)$ is bounded from $L^{2}(\mathcal{X}) \times L^{\infty}(\mathcal{X})$ into $L^{2}(\mathcal{X})$, which, combined with the fact that $L^{\infty}(\mathcal{X}) \subset \operatorname{BMO}(\mathcal{X})$ and some arguments used in the proof of (i) through (iv) of Theorem 3 , implies that the kernel of the operator $K_{g}^{(1)}(\cdot):=\Pi_{1}(\cdot, g)$, defined by setting, for any $(x, y) \in \mathcal{X} \times \mathcal{X}$,

$$
K_{g}^{(1)}(x, y):=\sum_{j \in \mathbb{Z}} P_{j}(x, y) Q_{j}(g)(x)
$$

satisfies (3) through (5), and hence $K_{g}^{(1)}$ is an $\eta$-Calderón-Zygmund operator which is bounded on $L^{2}(\mathcal{X})$. By these and the boundedness of Calderón-Zygmund operators (see, for example, ([27], Theorem 2.4 in Chapter III)), we finish the proof of (v) and (vi) of Theorem 4 and hence of Theorem 4.

Remark 9. We observe that the proofs of Theorems 3 and 4 do not use the second difference regularity condition of $\left\{Q_{j}\right\}_{j \in \mathbb{Z}}$ in Definition 4 . Thus, the results in Theorems 3 and 4 hold true for more general approximations of identity.

Author Contributions: Conceptualization, X.F.; methodology, X.F.; software, X.F.; validation, X.F.; formal analysis, X.F.; investigation, X.F.; resources, X.F.; data curation, X.F.; writing-original draft preparation, X.F.; writing-review and editing, X.F.; visualization, X.F.; supervision, X.F.; project administration, X.F.; funding acquisition, X.F. The author has read and agreed to the published version of the manuscript.

Funding: This research was funded by the National Natural Science Foundation of China (Grant Nos. 11701160 and 11871100).

Institutional Review Board Statement: Not applicable.

Informed Consent Statement: Not applicable.

Data Availability Statement: Not applicable.

Acknowledgments: We would like to express our deep thanks to the anonymous referees for their valuable comments, which improve the presentation of this paper.

Conflicts of Interest: The author declares no conflict of interest.

\section{References}

1. Fujita, H.; Kato, T. On the Navier-Stokes initial value problem. I. Arch. Rational Mech. Anal. 1964, 16, 269-315. [CrossRef]

2. Kato, T. Strong $L^{p}$-solutions of the Navier-Stokes equation in $\mathbb{R}^{m}$, with applications to weak solutions. Math. Z. 1984, 187, 471-480. [CrossRef] 
3. Meyer, Y.; Coifman, R.R. Opérateurs pseudo-différentiels et théorème de Calderón. In (French) Séminaire d'Analyse Harmonique (1976-1977); Publ. Math. Orsay, No. 77-77; Dépt. Math., Univ. Paris-Sud: Orsay, France, 1977; pp. $28-40$.

4. Meyer, Y.; Coifman, R.R. Au Delà des Opérateurs Pseudo-différentiels, (French) [Beyond Pseudodifferential Operators] With an English Summary, Astérisque 57; Société Mathématique de France: Paris, France, 1978.

5. Meyer, Y.; Coifman, R.R. Wavelets. In Calderón-Zygmund and Multilinear Operators, Translated from the 1990 and 1991 French Originals by David Salinger, Cambridge Studies in Advanced Mathematics 48; Cambridge University Press: Cambridge, UK, 1997.

6. Bony, J.-M. Calcul symbolique et propagation des singularités pour les équations aux dérivées partielles non linéaires, (French) [Symbolic calculus and propagation of singularities for nonlinear partial differential equations]. Ann. Sci. École Norm. Sup. 1981, 14, 209-246. [CrossRef]

7. Bényi, Á.; Maldonado, D.; Nahmod, A.R.; Torres, R.H. Bilinear paraproducts revisited. Math. Nachr. 2010, 283, 1257-1276. [CrossRef]

8. Bernicot, F. Uniform estimates for paraproducts and related multilinear multipliers. Rev. Mat. Iberoam. 2009, 25, 1055-1088. [CrossRef]

9. Bernicot, F. A T(1)-theorem in relation to a semigroup of operators and applications to new paraproducts. Trans. Am. Math. Soc. 2012, 364, 6071-6108. [CrossRef]

10. Bernicot, F. Fiber-wise Calderón-Zygmund decomposition and application to a bi-dimen-sional paraproduct. Ill. J. Math. 2012, $56,415-422$.

11. David, G.; Journé, J.-L. A boundedness criterion for generalized Calderón-Zygmund operators. Ann. Math. 1984, 120, 371-397. [CrossRef]

12. Gilbert, J.E.; Nahmod, A.R. Bilinear operators with non-smooth symbol. I. J. Fourier Anal. Appl. 2001, 7, 435-467. [CrossRef]

13. Gilbert, J.E.; Nahmod, A.R. $L^{p}$-boundedness for time-frequency paraproducts. II. J. Fourier Anal. Appl. 2002, 8, 109-172. [CrossRef]

14. Grafakos, L.; Kalton, N.J. The Marcinkiewicz multiplier condition for bilinear operators. Studia Math. 2001, 146, 115-156. [CrossRef]

15. Muscalu, C.; Pipher, J.; Tao, T.; Thiele, C. Bi-parameter paraproducts. Acta Math. 2004, 193, 269-296. [CrossRef]

16. Muscalu, C.; Tao, T.; Thiele, C. Uniform estimates on multi-linear operators with modulation symmetry. Dedicated to the memory of Tom Wolff. J. Anal. Math. 2002, 88, 255-309. [CrossRef]

17. Germain, P.; Masmoudi, N.; Shatah, J. Global solutions for the gravity water waves equation in dimension 3. Ann. Math. 2012, 175, 691-754. [CrossRef]

18. Germain, P.; Masmoudi, N.; Shatah, J. Global solutions for 2D quadratic Schrödinger equations. J. Math. Pures Appl. 2012, 97, 505-543. [CrossRef]

19. Grafakos, L.; Torres, R.H. Discrete decompositions for bilinear operators and almost diagonal conditions. Trans. Am. Math. Soc. 2002, 354, 1153-1176. [CrossRef]

20. Bonami, A.; Grellier, S.; Ky, L.D. Paraproducts and products of functions in $\operatorname{BMO}\left(\mathbb{R}^{n}\right)$ and $H^{1}\left(\mathbb{R}^{n}\right)$ through wavelets. J. Math Pures Appl. 2012, 97, 230-241. [CrossRef]

21. Ky, L.D. Bilinear decompositions for the product space $H_{L}^{1} \times \mathrm{BMO}_{L}$. Math. Nachr. 2014, 287, 1288-1297. [CrossRef]

22. Ky, L.D. Bilinear decompositions and commutators of singular integral operators. Trans. Am. Math. Soc. 2013, 365, 2931-2958. [CrossRef]

23. Ky, L.D. Endpoint estimates for commutators of singular integrals related to Schrödinger operators. Rev. Mat. Iberoam. 2015, 31, 1333-1373.

24. Bényi, Á.; Maldonado, D.; Naibo, V. What is ... a paraproduct? Notices Am. Math. Soc. 2010, 57, 858-860.

25. Muscalu, C.; Schlag, W. Classical and Multilinear Harmonic Analysis, Volume II; Cambridge Studies in Advanced Mathematics 138; Cambridge University Press: Cambridge, UK, 2013.

26. Yang, D.; Liang, Y.; Ky, L.D. Real-Variable Theory of Musielak-Orlicz Hardy Spaces; Lecture Notes in Mathematics 2182; Springer: Cham, Switzerland, 2017.

27. Coifman, R.R.; Weiss, G. Analyse Harmonique Non-Commutative sur Certains Espaces Homogènes, (French) Étude de Certaines Intégrales Singulières; Lecture Notes in Mathematics 242; Springer: Berlin, Germany; New York, NY, USA, 1971.

28. Coifman, R.R.; Weiss, G. Extensions of Hardy spaces and their use in analysis. Bull. Am. Math. Soc. 1977, 83, 569-645. [CrossRef]

29. Fu, X.; Yang, D.; Yang, S. Endpoint boundedness of linear commutators on local Hardy spaces over metric measure spaces of homogeneous type. J. Geom. Anal. 2020, 31, 4092-4164. [CrossRef]

30. Chang, D.-C.; Fu, X.; Yang, D. Boundedness of paraproducts on spaces of homogeneous type I. Appl. Anal. 2020. [CrossRef]

31. Nakai, E.; Yabuta, K. Pointwise multipliers for functions of weighted bounded mean oscillation on spaces of homogeneous type. Math. Japon. 1997, 46, 15-28.

32. Auscher, P.; Hytönen, T. Orthonormal bases of regular wavelets in spaces of homogeneous type. Appl. Comput. Harmon. Anal. 2013, 34, 266-296. [CrossRef]

33. Han, Y.; Müller, D.; Yang, D. A theory of Besov and Triebel-Lizorkin spaces on metric measure spaces modeled on CarnotCarathéodory spaces. Abstr. Appl. Anal. 2008, 2008, 893409. [CrossRef]

34. Han, Y.; Müller, D.; Yang, D. Littlewood-Paley characterizations for Hardy spaces on spaces of homogeneous type. Math. Nachr. 2006, 279, 1505-1537. [CrossRef] 
35. Deng, D.; Han, Y. Harmonic Analysis on Spaces of Homogeneous Type; Lecture Notes in Mathematics 1966; Springer: Berlin, Germany, 2009.

36. Macías, R.A.; Segovia, C. A decomposition into atoms of distributions on spaces of homogeneous type. Adv. Math. 1979, 33, 271-309. [CrossRef]

37. Bui, H.-Q.; Bui, T.A.; Duong, X.T. Weighted Besov and Triebel—Lizorkin spaces associated to operators and applications. Forum Math. Sigma 2020, 8, e11. [CrossRef]

38. Bui, T.A.; Duong, X.T. Sharp weighted estimates for square functions associated to operators on spaces of homogeneous type. J. Geom. Anal. 2020, 30, 874-900. [CrossRef]

39. Bui, T.A.; Duong, X.T.; Ky, L.D. Hardy spaces associated to critical functions and applications to T1 theorems. J. Fourier Anal. Appl. 2020, 26, 27. [CrossRef]

40. Bui, T.A.; Duong, X.T.; Ly, F.K. Maximal function characterizations for new local Hardy type spaces on spaces of homogeneous type. Trans. Am. Math. Soc. 2018, 370, 7229-7292. [CrossRef]

41. Bui, T.A.; Duong, X.T.; Ly, F.K. Maximal function characterizations for Hardy spaces on spaces of homogeneous type with finite measure and applications. J. Funct. Anal. 2020, 278, 108423. [CrossRef]

42. Grafakos, L.; Liu, L.; Maldonado, D.; Yang, D. Multilinear analysis on metric spaces. Diss. Math. 2014, 497, 1-121. [CrossRef]

43. Grafakos, L.; Liu, L.; Yang, D. Boundedness of paraproduct operators on RD-spaces. Sci. China Math. 2010, 53, 2097-2114. [CrossRef]

44. Hu, G.; Yang, D.; Zhou, Y. Boundedness of singular integrals in Hardy spaces on spaces of homogeneous type. Taiwan. J. Math. 2009, 13, 91-135. [CrossRef]

45. Yang, D.; Zhou, Y. Radial maximal function characterizations of Hardy spaces on RD-spaces and their applications. Math. Ann. 2010, 346, 307-333. [CrossRef]

46. Yang, D.; Zhou, Y. New properties of Besov and Triebel-Lizorkin spaces on RD-spaces. Manuscripta Math. 2011, 134, 59-90. [CrossRef]

47. Fu, X.; Ma, T.; Yang, D. Real-variable characterizations of Musielak-Orlicz Hardy spaces on spaces of homogeneous type. Ann. Acad. Sci. Fenn. Math. 2020, 45, 343-410. [CrossRef]

48. Fu, X.; Yang, D.; Liang, Y. Products of functions in $\operatorname{BMO}(\mathcal{X})$ and $H_{\mathrm{at}}^{1}(\mathcal{X})$ via wavelets over spaces of homogeneous type. J. Fourier Anal. Appl. 2017, 23, 919-990. [CrossRef]

49. Han, Y.; Han, Y.; He, Z.; Li, J.; Pereyra, C. Geometric characteriztions of embedding theorems—For Sobolev, Besov and Triebel— Lizorkin spaces on spaces of homogeneous type-Via orthonormal wavelets. J. Geom. Anal. 2021, 31, 8947-8978. [CrossRef]

50. Han, Y.; Han, Y.; Li, J. Criterion of the boundedness of singular integrals on spaces of homogeneous type. J. Funct. Anal. 2016, 271, 3423-3464. [CrossRef]

51. Han, Y.; Han, Y.; Li, J. Geometry and Hardy spaces on spaces of homogeneous type in the sense of Coifman and Weiss. Sci. China Math. 2017, 60, 2199-2218. [CrossRef]

52. Han, Y.; Li, J.; Ward, L.A. Hardy space theory on spaces of homogeneous type via orthonormal wavelet bases. Appl. Comput. Harmon. Anal. 2018, 45, 120-169. [CrossRef]

53. He, Z.; Han, Y.; Li, J.; Liu, L.; Yang, D.; Yuan, W. A complete real-variable theory of Hardy spaces on spaces of homogeneous type. J. Fourier Anal. Appl. 2019, 25, 2197-2267. [CrossRef]

54. He, Z.; Liu, L.; Yang, D.; Yuan, W. New Calderón reproducing formulae with exponential decay on spaces of homogeneous type. Sci. China Math. 2019, 62, 283-350. [CrossRef]

55. He, Z.; Wang, F.; Yang, D.; Yuan, W. Wavelet characterizations of Besov and Triebel—Lizorkin spaces on spaces of homogeneous type and their applications. Appl. Comput. Harmon. Anal. 2021, 54, 176-226. [CrossRef]

56. He, Z.; Yang, D.; Yuan, W. Real-variable characterizations of local Hardy spaces on spaces of homogeneous type. Math. Nachr. 2021, 294, 900-955. [CrossRef]

57. Liu, L.; Chang, D.-C.; Fu, X.; Yang, D. Endpoint boundedness of commutators on spaces of homogeneous type. Appl. Anal. 2017, 96, 2408-2433. [CrossRef]

58. Liu, L.; Chang, D.-C.; Fu, X.; Yang, D. Endpoint estimates of linear commutators on Hardy spaces over spaces of homogeneous type. Math. Meth. Appl. Sci. 2018, 41, 5951-5984. [CrossRef]

59. Liu, L.; Yang, D.; Yuan, W. Bilinear decompositions for products of Hardy and Lipschitz spaces on spaces of homogeneous type. Diss. Math. (Rozpr. Mat.) 2018, 533, 1-93. [CrossRef]

60. Wang, F.; Han, Y.; He, Z.; Yang, D. Besov spaces and Triebel-Lizorkin spaces on spaces of homogeneous type with their applications to a boundedness of Calderón-Zygmund operators. arXiv 2020, arXiv:2012.13035.

61. Zhou, X.; He, Z.; Yang, D. Real-variable characterizations of Hardy-Lorentz spaces on spaces of homogeneous type with applications to real interpolation and boundedness of Calderón-Zygmund operators. Anal. Geom. Metr. Spaces 2020, 8, 182-260. [CrossRef]

62. Chang, D.-C.; Fu, X.; Yang, D. Boundedness of paraproducts on spaces of homogeneous type II. Appl. Anal. 2020. [CrossRef]

63. Fu, X.; Chang, D.-C.; Yang, D. Recent progress in bilinear decompositions. Appl. Anal. Optim. 2017, 1, $153-210$.

64. Hytönen, T.; Kairema, A. Systems of dyadic cubes in a doubling metric space. Colloq. Math. 2012, 126, 1-33. [CrossRef]

65. Fu, X.; Yang, D. Wavelet characterizations of the atomic Hardy space $H^{1}$ on spaces of homogeneous type. Appl. Comput. Harmon. Anal. 2018, 44, 1-37. [CrossRef] 
66. Stein, E.M. Harmonic Analysis: Real-Variable Methods, Orthogonality and Oscillatory Integrals; Princeton University Press: Princeton, NJ, USA, 1993.

67. Grafakos, L. Modern Fourier Analysis, 3rd ed.; Graduate Texts in Mathematics 250; Springer: New York, NY, USA, 2014. 